\title{
WISHART LAWS AND VARIANCE FUNCTION ON HOMOGENEOUS CONES*
}

BY

\author{
PIOTR G RACZY K (ANGERS), HIDEYUKI IS H I** (NAGOYA AND KAWAGUCHI), \\ AND BARTOSZ KOŁODZIEJEK ${ }^{* * *}$ (WARSZAWA)
}

\begin{abstract}
We present a systematic study of Riesz measures and their natural exponential families of Wishart laws on a homogeneous cone. We compute explicitly the inverse of the mean map and the variance function of a Wishart exponential family.
\end{abstract}

2010 AMS Mathematics Subject Classification: Primary: 15B48; Secondary: 62H05.

Key words and phrases: Natural exponential families, variance function, Wishart laws, Riesz measure, homogeneous cones, graphical cones.

\section{INTRODUCTION}

Modern statistics and multivariate analysis require use of models on subcones of the cone $\operatorname{Sym}_{+}(n, \mathbb{R})$ of positive definite symmetric matrices. Such subcones are obtained for example by prescribing some of the off-diagonal elements to be zero. One considers two types of Wishart laws on such subcones [24]. The first type corresponds to the law of the maximum likelihood estimators of covariance matrices in a sample of size $n$ of a multidimensional normal vector $\left(X_{1}, \ldots, X_{p}\right)$ subject to conditional independence constraints (see [22], [24]). The inverses of the second type Wishart laws form a conjugate family of priors for the covariance parameter of the graphical Gaussian model. Some subclasses of second type Wishart laws, called G-Wishart in [24], are Diaconis-Ylvisaker conjugate priors (see [6]) for the precision matrix.

Many of such subcones are homogeneous, i.e. their automorphism group acts transitively. Recall that in the theory of graphical models [22], a decomposable graph generates a homogeneous cone if and only if the graph induced by any

* This research benefited from the support of the French government "Investissements d'Avenir" program ANR-11-LABX-0020-01 and PDLL Regional grant DéfiMaths.

** Partially supported by JSPS KAKENHI Grant Number 16K05174 and JST PRESTO.

*** Partially supported by NCN Grant no. 2012/05/B/ST1/0055. 
quadruple of vertices is not the graph $\bullet-\bullet-\bullet-\bullet$, denoted by $A_{4}$, cf. [16] and [24]. Among all decomposable graphs with four vertices, approximately 80 per cent are homogeneous.

Let us underline one more statistical motivation for developing the theory of Wishart laws on homogeneous cones. Modern big data statistics concentrates on the case $n \ll p$ in the multivariate normal sample of the vector $\left(X_{1}, \ldots, X_{p}\right)$, cf. [13]. This case corresponds to singular Wishart laws, which at the moment are fully described only on homogeneous cones, cf. Theorems 4.0 and 4.2 below.

Moreover, important recent statistical articles [24], [21] point out the significance of homogeneous cones among cones corresponding to decomposable and DAG models and devote much space to multivariate analysis on homogeneous cones. Further, Wishart laws on homogeneous cones supply many good examples of exponential families with very explicit calculation. This should be emphasized as a raison d'être of our research.

The preponderant role of homogeneous cones among subcones of $\operatorname{Sym}_{+}(n, \mathbb{R})$ strongly motivates research on Wishart laws on general homogeneous cones. Families of Wishart laws on homogeneous cones were first studied by Andersson and Wojnar [2], Boutouria [4] and Letac and Massam [24]. The pioneering paper [2] is very technical and inaccessible due to the use of methods based on Vinberg algebras. The natural approach to this topic, based on quadratic maps and matrix realizations of homogeneous cones, was proposed in Graczyk and Ishi [9], [17] and used in Ishi and Kołodziejek [20].

This article is a continuation of [9], [17] and [20], however here we identify the dual space using the trace inner product instead of the standard inner product. Indeed, the trace inner product will be indispensable in future applications to statistics. Though the difference between standard and trace inner products is rather technical, it gives rise to some changes in formulas in our previous works about generalized power functions and Wishart exponential families, i.e. the natural exponential families generated by Riesz measures. Thus we repeat and simplify some definitions and proofs from [9] and [17] for completeness and convenience of the reader. We hope that, eventually, this paper presents our methods and results in a manner which is accessible to mathematical statisticians.

The variance function is an important characteristic of a natural exponential family, cf. [3]. Let us consider a classical Wishart exponential family on the cone $\operatorname{Sym}_{+}(n, \mathbb{R})$, i.e. the natural exponential family (NEF) generated by a Riesz measure $\mu_{p}$ on $\operatorname{Sym}_{+}(n, \mathbb{R})$, with the Laplace transform $L_{\mu_{p}}(\theta)=(\operatorname{det} \theta)^{-p}$ for $\theta \in \operatorname{Sym}_{+}(n, \mathbb{R})$. Here $p$ belongs to the Gindikin-Wallach set (in this context also called the Jorgensen set $) \Lambda=\{1 / 2,1,3 / 2, \ldots,(n-1) / 2\} \cup((n-1) / 2, \infty)$. It is well known and straightforward to check (see, for example, [23]) that the variance function of NEF generated by $\mu_{p}$ is given by

$$
\mathbb{V}_{p}(m)=\frac{1}{p} \rho(m)
$$


where $m \in\left(\operatorname{Sym}_{+}(n, \mathbb{R})\right)^{*} \equiv \operatorname{Sym}_{+}(n, \mathbb{R})$ and $\rho(m)$ is a linear map from $\operatorname{Sym}(n, \mathbb{R})$ to itself defined by $\rho(m) Y=m Y m^{\top}, Y \in \operatorname{Sym}(n, \mathbb{R})$. Generally, the Wishart exponential family is the natural exponential family generated by some Riesz measure. In this paper we give explicit formulas for the variance function of Wishart exponential family on any homogeneous cone.

Let us describe shortly the plan of this paper. In Section $\square$ we recall the definition of a natural exponential family generated by a positive measure and we introduce their characteristics, mean and variance, used and studied throughout the whole paper.

Sections $[3$ and 4 are devoted to introducing the main tools for the analysis of Wishart exponential families on homogeneous cones. Like in [9], we consider two types of homogeneous cones $-\mathcal{P}_{\mathcal{V}}$ which is in a matrix realization and its dual cone $\mathcal{Q V}_{\mathcal{V}}$ - and Riesz measures and Wishart families on them. This corresponds to the concept of Type I and Type II Wishart laws defined and studied in [24].

Any homogeneous cone is linearly isomorphic to some matrix cone $\mathcal{P}_{\mathcal{V}}$ (see [18]). It was observed in [9] that the matrix realization of a homogeneous cone makes analysis of Riesz and Wishart measures much easier. The present paper is also based on this technique, which is reviewed in Section B.D.

In Section B.2, we define the generalized power functions $\delta_{\underline{s}}$ and $\Delta_{\underline{s}}$ on the cones $\mathcal{Q}_{\mathcal{V}}$ and $\mathcal{P}_{\mathcal{V}}$, respectively. In Proposition B.J(iii), we give a formula, i.e. (B.7), for the power function $\delta_{\underline{s}}$, which is new and useful. In Definition 3.3 we introduce an important map $\xi \mapsto \hat{\xi}$ between $\mathcal{Q}_{\mathcal{V}}$ and $\operatorname{Sym}_{+}(N, \mathbb{R})$, inspired by an analogous map playing a fundamental role for decomposable graphical models [22].

In Section [1, we first prove formula (5.1), which serves as a simple and useful tool in our argument. Then we deduce from (5.1) an explicit evaluation of the inverse of the mean map (Theorem 5.1). It allows us to find the Lauritzen formula on any (not only graphical) homogeneous cone. We get in Section 6 the variance function formula for Wishart exponential families defined on the cone $\mathcal{Q}_{\mathcal{V}}$. The proof is based on formula (5.1) again. Note that Riesz and Wishart measures on cones $Q_{G}$ and $P_{G}$ corresponding to the decomposable graphs $G$ were studied in [24] and (uniquely in case $Q_{G}$ ) in [I]. When the cones $Q_{G}$ and $P_{G}$ are homogeneous, the integral formulas in Theorems 3.1 and 3.2 of [24] and in Theorem 5.1 of [I] are a special case of our results on the cones $\mathcal{Q}_{\mathcal{V}}$ and $\mathcal{P} \mathcal{V}$, when the dimensions of all the blocks in the matrix realization of $\mathcal{Q}_{\mathcal{V}}$ are equal to one.

In Section $\square$ we give results on the variance function formula for Wishart exponential families on $\mathcal{P}_{\mathcal{V}}$. In particular, we offer a practical approach to the construction of a matrix realization of the dual cone $\mathcal{Q}_{\mathcal{V}}$, using basic quadratic maps. Note that if a homogeneous cone $\mathcal{P}_{\mathcal{V}}$ is in matrix realization, then, in general, $\mathcal{Q}_{\mathcal{V}}$ is not, which makes analysis on $\mathcal{Q} \mathcal{V}$ harder.

The last Section 8 contains applications of the results and of the methods of this paper to symmetric cones and graphical homogeneous cones. We improve and complete in this way the results of [12] and [24]. 
The unpublished paper [5] may seem to contain results of our paper. However, the main results of [5], announced in Theorems 3.3 and 4.2, are false. The proofs of these theorems are based on several erroneous arguments. Consequently, the unpublished paper [5] cannot be compared with the results of our paper.

The full version of the Introduction and the exposition of errors in [5] were accepted by the referee and are available on arXiv [10]. On the demand of the editor, we present a shortened version of the paper.

Acknowledgments. We thank Małgorzata Bogdan and Hélène Massam for discussions on statistical applications of Wishart laws on homogeneous cones.

\section{NATURAL EXPONENTIAL FAMILIES}

In the following section we will give a short introduction to natural exponential families (NEFs). The standard reference book on exponential families is [3].

Let $\mathbb{E}$ be a finite-dimensional real linear space endowed with an inner product $\langle\cdot, \cdot\rangle$ and let $\mathbb{E}^{*}$ be the dual space of $\mathbb{E}$. If $\xi \in \mathbb{E}^{*}$ is a linear functional on $\mathbb{E}$, we will denote its action on $x \in \mathbb{E}$ by $\langle\xi, x\rangle$. Let $L_{S}\left(\mathbb{E}^{*}, \mathbb{E}\right)$ be the linear space of linear operators $A: \mathbb{E}^{*} \rightarrow \mathbb{E}$ such that for any $\xi, \eta \in \mathbb{E}^{*}$, one has $\langle\xi, A(\eta)\rangle=\langle\eta, A(\xi)\rangle$.

Let $\mu$ be a positive Radon measure on $\mathbb{E}$. We define its Laplace transform $L_{\mu}: \mathbb{E}^{*} \rightarrow(0, \infty]$ by

$$
L_{\mu}(\theta):=\int_{\mathbb{E}} e^{-\langle\theta, x\rangle} \mu(d x) .
$$

Let $\Theta(\mu)$ denote the interior of the set $\left\{\theta \in \mathbb{E}^{*}: L_{\mu}(\theta)<\infty\right\}$. Hölder's inequality implies that the set $\Theta(\mu)$ is convex and the cumulant function

$$
k_{\mu}(\theta):=\log L_{\mu}(\theta)
$$

is convex on $\Theta(\mu)$ and it is strictly convex if and only if $\mu$ is not concentrated on any affine hyperplane of $\mathbb{E}$. Let $\mathcal{M}(\mathbb{E})$ be the set of positive Radon measures on $\mathbb{E}$ such that $\Theta(\mu)$ is not empty and $\mu$ is not concentrated on any affine hyperplane of $\mathbb{E}$.

For $\mu \in \mathcal{M}(\mathbb{E})$ we define the natural exponential family (NEF) generated by $\mu$ as the set of probability measures

$$
F(\mu)=\left\{P(\theta, \mu)(d x)=e^{-\langle\theta, x\rangle-k_{\mu}(\theta)} \mu(d x): \theta \in \Theta(\mu)\right\} .
$$

Then, for $\theta \in \Theta(\mu)$,

$$
\begin{aligned}
m_{\mu}(\theta) & :=-k_{\mu}^{\prime}(\theta)=\int_{\mathbb{E}} x P(\theta, \mu)(d x), \\
-m_{\mu}^{\prime}(\theta) & =k_{\mu}^{\prime \prime}(\theta)=\int_{\mathbb{E}}\left(x-m_{\mu}(\theta)\right) \otimes\left(x-m_{\mu}(\theta)\right) P(\theta, \mu)(d x)
\end{aligned}
$$

are respectively the mean and the covariance operator of the measure $P(\theta, \mu)$. Here $x \otimes x$ is an element of $L_{S}\left(\mathbb{E}^{*}, \mathbb{E}\right)$ defined by $(x \otimes x)(\xi)=x\langle\xi, x\rangle$ for $x \in \mathbb{E}$ and 
$\xi \in \mathbb{E}^{*}$. The subset $M_{F(\mu)}:=m_{\mu}(\Theta(\mu))$ of $\mathbb{E}$ is called the domain of means of $F(\mu)$. The map $m_{\mu}: \Theta(\mu) \rightarrow M_{F(\mu)}$ is an analytic diffeomorphism, and its inverse is denoted by $\psi_{\mu}: M_{F(\mu)} \rightarrow \Theta(\mu)$.

Lemma 2.1 ([17], Proposition IV.4). Define

$$
J_{\mu}(m):=\sup _{\theta \in \Theta(\mu)} \frac{e^{-\langle\theta, m\rangle}}{L_{\mu}(\theta)} \quad \text { for any } m \in M_{F(\mu)} .
$$

Then $\psi_{\mu}=-\left(\log J_{\mu}\right)^{\prime}$.

Proof. Since $\log J_{\mu}(-m)=\sup _{\theta \in \Theta(\mu)}\left(\langle\theta, m\rangle-k_{\mu}(\theta)\right)$ is the LegendreFenchel transform of $k_{\mu}(\theta)$, the statement follows from the Fenchel duality.

For any $m \in M_{F(\mu)}$ consider the covariance operator $\mathbb{V}_{F(\mu)}(m)$ of the measure $P\left(\psi_{\mu}(m), \mu\right)$. Then

$$
\mathbb{V}_{F(\mu)}(m)=k_{\mu}^{\prime \prime}\left(\psi_{\mu}(m)\right)=-\left[\psi_{\mu}^{\prime}(m)\right]^{-1} .
$$

The map $\mathbb{V}_{F(\mu)}: M_{F(\mu)} \rightarrow L_{S}\left(\mathbb{E}^{*}, \mathbb{E}\right)$ is called the variance function of $F(\mu)$. The variance function is a central object of interest of natural exponential families, because it characterizes an NEF and the generating measure in the following way: if $F(\mu)$ and $F\left(\mu_{0}\right)$ are two natural exponential families such that $\mathbb{V}_{F(\mu)}$ and $\mathbb{V}_{F\left(\mu_{0}\right)}$ coincide on a non-void open set $J \subset M_{F(\mu)} \cap M_{F\left(\mu_{0}\right)}$, then $F(\mu)=F\left(\mu_{0}\right)$ and so $\mu_{0}(d x)=\exp \{\langle a, x\rangle+b\} \mu(d x)$ for some $a \in \mathbb{E}^{*}$ and $b \in \mathbb{R}$. The variance function gives full knowledge of the NEF.

In the context of natural exponential families, invariance properties under the action of a subgroup of general linear group or general affine group are often considered. For the recent developments in this direction see [20].

\section{BASIC FACTS ON HOMOGENEOUS CONES}

Let $\operatorname{Mat}(n, m ; \mathbb{R})$ and $\operatorname{Sym}(n, \mathbb{R})$ denote the linear spaces of real $n \times m$ matrices and symmetric real $n \times n$ matrices, respectively. Let $\operatorname{Sym}_{+}(n, \mathbb{R})$ be the cone of symmetric positive definite real $n \times n$ matrices. $A^{\top}$ denotes the transpose of a matrix $A$. For $X \in \operatorname{Mat}(N, N ; \mathbb{R})$ define a linear operator $\rho(X): \operatorname{Sym}(N, \mathbb{R}) \rightarrow$ $\operatorname{Sym}(N, \mathbb{R})$ by $\rho(X) Y=X Y X^{\top}, Y \in \operatorname{Sym}(N, \mathbb{R})$.

Let $V$ be a real linear space and $\Omega$ a regular open convex cone in $V$. The open convex cone $\Omega$ is regular if $\bar{\Omega} \cap(-\bar{\Omega})=\{0\}$. The linear automorphism group preserving the cone is denoted by $\mathrm{G}(\Omega)=\{g \in G L(V): g \Omega=\Omega\}$. The cone $\Omega$ is said to be homogeneous if $\mathrm{G}(\Omega)$ acts transitively on $\Omega$.

3.1. Homogeneous cones $\mathcal{P}_{\mathcal{V}}$ and $\mathcal{Q}_{\mathcal{V}}$. We recall from [9] a useful realization of any homogeneous cone. Let us take a partition $N=n_{1}+\ldots+n_{r}$ of a positive integer $N$ and consider a system of vector spaces $\mathcal{V}_{l k} \subset \operatorname{Mat}\left(n_{l}, n_{k} ; \mathbb{R}\right)$, $1 \leqslant k<l \leqslant r$, satisfying the following three conditions: 
(V1) $A \in \mathcal{V}_{l k}, B \in \mathcal{V}_{k i} \Longrightarrow A B \in \mathcal{V}_{l i}$ for any $1 \leqslant i<k<l \leqslant r$;

(V2) $A \in \mathcal{V}_{l i}, B \in \mathcal{V}_{k i} \Longrightarrow A B^{\top} \in \mathcal{V}_{l k}$ for any $1 \leqslant i<k<l \leqslant r$;

(V3) $A \in \mathcal{V}_{l k} \Longrightarrow A A^{\top} \in \mathbb{R} I_{n_{l}}$ for any $1 \leqslant k<l \leqslant r$.

Let $\mathcal{Z}_{\mathcal{V}}$ be the subspace of $\operatorname{Sym}(N, \mathbb{R})$ defined by

$$
\mathcal{Z}_{\mathcal{V}}:=\left\{x=\left(\begin{array}{cccc}
X_{11} & X_{21}^{\top} & \ldots & X_{r 1}^{\top} \\
X_{21} & X_{22} & \ldots & X_{r 2}^{\top} \\
\vdots & & \ddots & \\
X_{r 1} & X_{r 2} & & X_{r r}
\end{array}\right): \begin{array}{l}
X_{l k} \in \mathcal{V}_{l k}, 1 \leqslant k<l \leqslant r, \\
X_{l l}=x_{l l} I_{n_{l}}, x_{l l} \in \mathbb{R}, 1 \leqslant l \leqslant r
\end{array}\right\} .
$$

We set

$$
\mathcal{P}_{\mathcal{V}}:=\mathcal{Z}_{\mathcal{V}} \cap \operatorname{Sym}_{+}(N, \mathbb{R})
$$

Then $\mathcal{P}_{\mathcal{V}}$ is a regular open convex cone in the linear space $\mathcal{Z}_{\mathcal{V}}$. Let $H_{\mathcal{V}}$ be the group of real lower triangular matrices with positive diagonals defined by

$$
H_{\mathcal{V}}:=\left\{T=\left(\begin{array}{cccc}
T_{11} & & & \\
T_{21} & T_{22} & & \\
\vdots & & \ddots & \\
T_{r 1} & T_{r 2} & & T_{r r}
\end{array}\right): \begin{array}{l}
T_{l k} \in \mathcal{V}_{l k}, 1 \leqslant k<l \leqslant r \\
T_{l l}=t_{l l} I_{n_{l}}, t_{l l}>0,1 \leqslant l \leqslant r
\end{array}\right\} .
$$

If $T \in H_{\mathcal{V}}$ and $x \in \mathcal{Z}_{\mathcal{V}}$, then $\rho(T) x=T x T^{\top} \in \mathcal{Z}_{\mathcal{V}}$ thanks to (V1)-(V3). Moreover, $\rho\left(H_{\mathcal{V}}\right)$ acts on the cone $\mathcal{P}_{\mathcal{V}}$ (simply) transitively ([15], Proposition 3.2), that is, $\mathcal{P}_{\mathcal{V}}$ is a homogeneous cone. Our interest in $\mathcal{P}_{\mathcal{V}}$ is motivated by the fact that any homogeneous cone is linearly isomorphic to $\mathcal{P}_{\mathcal{V}}$ due to Theorem $\mathrm{D}$ in [15]].

Condition (V3) allows us to define an inner product $(\cdot \mid \cdot)$ on $\mathcal{V}_{l k}, 1 \leqslant k<l \leqslant r$, by the formula

$$
\left(A B^{\top}+B A^{\top}\right) / 2=(A \mid B) I_{n_{l}}, \quad A, B \in \mathcal{V}_{l k} .
$$

We define the trace inner product on $\mathcal{Z}_{\mathcal{V}}$ by

$$
\langle x, y\rangle=\operatorname{tr}(x y)=\sum_{k=1}^{r} n_{k} x_{k k} y_{k k}+2 \sum_{1 \leqslant k<l \leqslant r} n_{l}\left(X_{l k} \mid Y_{l k}\right), \quad x, y \in \mathcal{Z}_{\mathcal{V}} .
$$

Using the trace inner product, we identify the dual space $\mathcal{Z}_{\mathcal{V}}^{*}$ with $\mathcal{Z}_{\mathcal{V}}$. Define the dual cone $\mathcal{Q} \mathcal{V}$ by

$$
\mathcal{Q}_{\mathcal{V}}:=\left\{\xi \in \mathcal{Z}_{\mathcal{V}}:\langle\xi, x\rangle>0 \forall x \in \overline{\mathcal{P}_{\mathcal{V}}} \backslash\{0\}\right\},
$$

where $\overline{\mathcal{P}_{\mathcal{V}}}$ is the closure of $\mathcal{P}_{\mathcal{V}}$. The dual cone $\mathcal{Q}_{\mathcal{V}}$ is also homogeneous. It is easily seen that $I_{N} \in \mathcal{Q}_{\mathcal{V}}$.

For $T \in H_{\mathcal{V}}$, we denote by $\rho^{*}(T)$ the adjoint operator of $\rho(T) \in G L\left(\mathcal{Z}_{\mathcal{V}}\right)$ defined in such a way that $\langle\xi, \rho(T) x\rangle=\left\langle\rho^{*}(T) \xi, x\right\rangle$ for any $\xi, x \in \mathcal{Z}_{\mathcal{V}}$. For any $\xi \in \mathcal{Q}_{\mathcal{V}}$ there exists a unique $T \in H_{\mathcal{V}}$ such that $\xi=\rho^{*}(T) I_{N}$ ([25], Chapter 1, Proposition 9). 
3.2. Generalized power functions. Define a one-dimensional representation $\chi_{\underline{s}}$ of the triangular group $H_{\mathcal{V}}$ by

$$
\chi_{\underline{s}}(T):=\prod_{k=1}^{r} t_{k k}^{2 s_{k}},
$$

where $\underline{s}=\left(s_{1}, \ldots, s_{r}\right) \in \mathbb{C}^{r}$. Note that any one-dimensional representation $\chi$ of $H_{\mathcal{V}}$ is of the form $\chi_{\underline{s}}$ for some $\underline{s} \in \mathbb{C}^{r}$.

Definition 3.1. Let $\Delta_{\underline{s}}: \mathcal{P}_{\mathcal{V}} \rightarrow \mathbb{C}$ be the function given by

$$
\Delta_{\underline{s}}\left(\rho(T) I_{N}\right):=\chi_{\underline{s}}(T), \quad T \in H_{\mathcal{V}} .
$$

Let $\delta_{\underline{s}}: \mathcal{Q}_{\mathcal{V}} \rightarrow \mathbb{C}$ be the function given by

$$
\delta_{\underline{s}}\left(\rho^{*}(T) I_{N}\right):=\chi_{\underline{s}}(T), \quad T \in H_{\mathcal{V}} .
$$

The functions $\Delta$ and $\delta$ are called generalized power functions.

Let $N_{k}=n_{1}+\ldots+n_{k}, k=1, \ldots, r$. For $y \in \operatorname{Sym}(N, \mathbb{R})$, by $y_{\{1: k\}} \in$ $\operatorname{Sym}\left(N_{k}, \mathbb{R}\right)$ we denote the submatrix $\left(y_{i j}\right)_{1 \leqslant i, j \leqslant N_{k}}$. It is known that for any lower triangular matrix $T$ one has $\left(T T^{\top}\right)_{\{1: k\}}=T_{\{1: k\}} T_{\{1: k\}}^{\top}$. Thus, for $x=\rho(T) I_{N} \in$ $\mathcal{P}_{\mathcal{V}}$ with $T \in H_{\mathcal{V}}$ one has $\operatorname{det} x_{\{1: k\}}=\left(\operatorname{det} T_{\{1: k\}}\right)^{2}=\prod_{i=1}^{k} t_{i i}^{2 n_{i}}$. This implies that for any $x \in \mathcal{P}_{\mathcal{V}}$,

$$
\Delta_{\underline{s}}(x)=(\operatorname{det} x)^{s_{r} / n_{r}} \prod_{k=1}^{r-1}\left(\operatorname{det} x_{\{1: k\}}\right)^{s_{k} / n_{k}-s_{k+1} / n_{k+1}} .
$$

We will express $\delta_{\underline{s}}(\xi)$ as a function of $\xi \in \mathcal{Q}_{\mathcal{V}}$ in Section 3.3 (see Proposition B.]). By definition, $\Delta_{\underline{s}}$ and $\delta_{\underline{s}}$ are multiplicative in the following sense:

$$
\begin{aligned}
& \Delta_{\underline{s}}(\rho(T) x)=\Delta_{\underline{s}}\left(\rho(T) I_{N}\right) \Delta_{\underline{s}}(x), \quad(x, T) \in \mathcal{P}_{\mathcal{V}} \times H_{\mathcal{V}}, \\
& \delta_{\underline{s}}\left(\rho^{*}(T) \xi\right)=\delta_{\underline{s}}\left(\rho^{*}(T) I_{N}\right) \delta_{\underline{s}}(\xi), \quad(\xi, T) \in \mathcal{Q}_{\mathcal{V}} \times H_{\mathcal{V}} .
\end{aligned}
$$

DEFINITION 3.2. Let $\pi: \operatorname{Sym}(N, \mathbb{R}) \rightarrow \mathcal{Z}_{\mathcal{V}}$ be the projection such that, for any $x \in \operatorname{Sym}(N, \mathbb{R})$, the element $\pi(x) \in \mathcal{Z}_{\mathcal{V}}$ is uniquely determined by

$$
\operatorname{tr}(x a)=\langle\pi(x), a\rangle, \quad \forall a \in \mathcal{Z}_{\mathcal{V}} .
$$

For any $x, y \in \mathcal{Z}_{\mathcal{V}}$ one has

$$
\left\langle\rho^{*}(T) x, y\right\rangle=\langle x, \rho(T) y\rangle=\operatorname{tr}\left(\rho\left(T^{\top}\right) x \cdot y\right)=\left\langle\pi\left(\rho\left(T^{\top}\right) x\right), y\right\rangle,
$$

thus, for any $T \in H_{\mathcal{V}}$,

$$
\rho^{*}(T)=\pi \circ \rho\left(T^{\top}\right) .
$$

Now we define a useful map $\xi \mapsto \hat{\xi}$ between $\mathcal{Q}_{\mathcal{V}}$ and $\operatorname{Sym}_{+}(N, \mathbb{R})$ such that $(\hat{\xi})^{-1} \in \mathcal{P} \mathcal{V}$ and $\pi(\hat{\xi})=\xi$. An analogous map is very important in statistics on decomposable graphical models [22]. 
Definition 3.3. For $\xi=\rho^{*}(T) I_{N} \in \mathcal{Q}_{\mathcal{V}}$ with $T \in H_{\mathcal{V}}$, we define

$$
\hat{\xi}:=\rho\left(T^{\top}\right) I_{N}=T^{\top} T \in \operatorname{Sym}_{+}(N, \mathbb{R}) .
$$

Note that for any $\xi \in \mathcal{Q} \mathcal{V}$, one has $(\hat{\xi})^{-1} \in \mathcal{P}_{\mathcal{V}}$ (compare the definition of $\hat{\xi}$ in [24], Proposition 2.1). Indeed, $(\hat{\xi})^{-1}=\rho\left(T^{-1}\right) I_{N} \in \mathcal{P} \mathcal{V}$. Due to (B.4), we have $\pi(\hat{\xi})=\xi$.

Observe that for $T \in H_{\mathcal{V}}$

$$
\Delta_{\underline{s}}\left(\rho(T) I_{N}\right)=\chi_{\underline{s}}(T)=\chi_{-\underline{s}}\left(T^{-1}\right)=\delta_{-\underline{s}}\left(\rho^{*}\left(T^{-1}\right) I_{N}\right)
$$

and, due to (3.4), $\rho^{*}\left(T^{-1}\right) I_{N}=\pi\left(\left(\rho(T) I_{N}\right)^{-1}\right)$. This implies that the functions $\Delta$ and $\delta$ are related by the following identities:

$$
\begin{aligned}
\Delta_{\underline{s}}(x) & =\delta_{-\underline{s}}\left(\pi\left(x^{-1}\right)\right), & & x \in \mathcal{P} \mathcal{V}, \\
\delta_{-\underline{s}}(\xi) & =\Delta_{\underline{s}}\left(\hat{\xi}^{-1}\right), & & \xi \in \mathcal{Q}_{\mathcal{V}} .
\end{aligned}
$$

In literature, the function $\delta_{\underline{s}}$ is sometimes denoted by $\Delta_{s^{*}}^{*}$, where $\underline{s}^{*}=\left(s_{r}, \ldots, s_{1}\right)$.

3.3. Basic quadratic maps $q_{i}$ and associated maps $\phi_{i}$. We recall from [9] a construction of basic quadratic maps. Let $W_{i}, i=1, \ldots, r$, be the subspace of $\operatorname{Mat}\left(N, n_{i} ; \mathbb{R}\right)$ consisting of the matrices $x$ of the form

$$
x=\left(0_{n_{i}, n_{1}+\ldots+n_{i-1}}, x_{i i} I_{n_{i}}, \ldots, X_{r i}^{\top}\right)^{\top},
$$

where $X_{l i} \in \mathcal{V}_{l i}, l=i+1, \ldots, r$. For $x \in W_{i}$, the symmetric matrix $x x^{\top}$ belongs to $\mathcal{Z}_{\mathcal{V}}$ thanks to (V2) and (V3). We define the basic quadratic map $q_{i}: W_{i} \ni x \mapsto$ $x x^{\top} \in \mathcal{Z}_{\mathcal{V}}$.

Taking an orthonormal basis of each $\mathcal{V}_{l i}$ with respect to $(\cdot \mid \cdot)$, we identify the space $W_{i}$ with $\mathbb{R}^{m_{i}}$, where $m_{i}=\operatorname{dim} W_{i}=1+\operatorname{dim} \mathcal{V}_{i+1, i}+\ldots+\operatorname{dim} \mathcal{V}_{r i}$. Let $\operatorname{vec}(x) \in \mathbb{R}^{m_{i}}$ denote the vectorization of $x \in W_{i}$. It is convenient to choose a basis for $W_{i}$ consistent with the block decomposition of $\mathcal{Z}_{\mathcal{V}}$, that is, $\left(v_{1}, \ldots, v_{m_{i}}\right)$, where $v_{1}$ corresponds to $\mathcal{V}_{i i} \simeq \mathbb{R}$ and $\left(v_{2}, \ldots, v_{1+\operatorname{dim}} \mathcal{V}_{i+1, i}\right)$ corresponds to $\mathcal{V}_{i+1, i}$, and so on.

DEFINITION 3.4. For the quadratic map $q_{i}$ we define the associated linear $\operatorname{map} \phi_{i}: \mathcal{Z}_{\mathcal{V}} \equiv \mathcal{Z}_{\mathcal{V}}^{*} \rightarrow \operatorname{Sym}\left(m_{i}, \mathbb{R}\right)$ in such a way that for $\xi \in \mathcal{Z}_{\mathcal{V}}$

$$
\operatorname{vec}(x)^{\top} \phi_{i}(\xi) \operatorname{vec}(x)=\left\langle\xi, q_{i}(x)\right\rangle, \quad \forall x \in W_{i} .
$$

Similarly we consider another subspace of $\operatorname{Mat}\left(N, n_{i} ; \mathbb{R}\right)$, namely,

$$
\widetilde{W}_{i}=\left\{x=\left(0_{n_{i}, n_{1}+\ldots+n_{i}}, X_{i+1, i}^{\top}, \ldots, X_{r i}^{\top}\right)^{\top}: X_{l i} \in \mathcal{V}_{l i}, l=i+1, \ldots, r\right\},
$$

the quadratic map $\breve{q}_{i}: \bar{W}_{i} \ni x \mapsto x x^{\top} \in \mathcal{Z}_{\mathcal{V}}$ and its associated linear map $\check{\phi}_{i}: \mathcal{Z}_{\mathcal{V}} \equiv \mathcal{Z}_{\mathcal{V}}^{*} \rightarrow \operatorname{Sym}\left(m_{i}-1, \mathbb{R}\right)$. 
Proposition 3.1. (i) For any $\xi \in \mathcal{Z}_{\mathcal{V}}$ and $i=1, \ldots, r-1$, one has

$$
\phi_{i}(\xi)=\left(\begin{array}{cc}
n_{i} \xi_{i i} & v_{i}(\xi)^{\top} \\
v_{i}(\xi) & \check{\phi}_{i}(\xi)
\end{array}\right),
$$

where $v_{i}(\xi)^{\top}:=\left(n_{i+1} \operatorname{vec}\left(\xi_{i+1, i}\right)^{\top}, \ldots, n_{r} \operatorname{vec}\left(\xi_{r i}\right)^{\top}\right) \in \mathbb{R}^{m_{i}-1}$ and $\operatorname{vec}\left(\xi_{k i}\right) \in$ $\mathbb{R}^{\operatorname{dim} \mathcal{V}_{k i}}$ is the vectorization of $\xi_{k i} \in \mathcal{V}_{k i}$. Moreover,

$$
\phi_{r}(\xi)=n_{r} \xi_{r r} \in \mathbb{R} \equiv \operatorname{Sym}(1, \mathbb{R}) .
$$

(ii) For $\xi=\rho^{*}(T) I_{N} \in \mathcal{Q}_{\mathcal{V}}$ with $T \in H_{\mathcal{V}}$ and $i=1, \ldots, r-1$, one has

$$
\begin{aligned}
& \operatorname{det} \phi_{i}(\xi)=\chi_{\underline{m}_{i}}(T) \operatorname{det} \phi_{i}\left(I_{N}\right), \\
& \operatorname{det} \check{\phi}_{i}(\xi)=\chi_{\underline{\underline{\underline{m}}}_{i}}(T) \operatorname{det} \check{\phi}_{i}\left(I_{N}\right),
\end{aligned}
$$

where

$$
\begin{aligned}
& \underline{\underline{m}}_{i}:=\left(0, \ldots, 0,1, n_{i+1, i}, \ldots, n_{r i}\right) \in \mathbb{Z}^{r}, \\
& \underline{\underline{m}}_{i}:=\left(0, \ldots, 0,0, n_{i+1, i}, \ldots, n_{r i}\right) \in \mathbb{Z}^{r} .
\end{aligned}
$$

(iii) For any $\xi \in \mathcal{Q}_{\mathcal{V}}$, one has

$$
\delta_{\underline{s}}(\xi)=C_{\underline{s}} \phi_{r}(\xi)^{s_{r}} \prod_{i=1}^{r-1}\left(\frac{\operatorname{det} \phi_{i}(\xi)}{\operatorname{det} \check{\phi}_{i}(\xi)}\right)^{s_{i}},
$$

where the constant $C_{\underline{s}}$ does not depend on $\xi$.

(iv) For $\alpha, \beta \in \mathcal{Z}_{\mathcal{V}}$ and $i=1, \ldots, r-1$, one has

$$
\begin{array}{r}
\operatorname{tr}\left(\phi_{i}(\alpha) \phi_{i}\left(I_{N}\right)^{-1} \phi_{i}(\beta) \phi_{i}\left(I_{N}\right)^{-1}\right)-\operatorname{tr}\left(\check{\phi}_{i}(\alpha) \check{\phi}_{i}\left(I_{N}\right)^{-1} \breve{\phi}_{i}(\beta) \check{\phi}_{i}\left(I_{N}\right)^{-1}\right) \\
=\alpha_{i i} \beta_{i i}+2 \sum_{l=i+1}^{r} \frac{n_{l}}{n_{i}}\left(\alpha_{l i} \mid \beta_{l i}\right) .
\end{array}
$$

Let us underline that the useful formula (B.7) for the power function $\delta_{\underline{s}}$ is new and different from the formula given in [9] and [17]. Precisely, it is just mentioned in [9], [17] that $\delta_{\underline{s}}(\xi)$ is a product of powers of $\operatorname{det} \phi_{i}(\xi)$.

Pr o of. (i) It is a consequence of the choice of basis for $W_{i}$ and the fact that $W_{i} \simeq \mathbb{R} \oplus \widetilde{W}_{i}$.

(ii) In [9] a very similar problem was considered, but there the dual space $\mathcal{Z}_{\mathcal{V}}^{*}$ was identified with $\mathcal{Z}_{\mathcal{V}}$ by using the so-called standard inner product rather than the trace inner product. The only difference in the form of $\phi_{i}$ in these two cases is that here block sizes $n_{i}$ appear in the $(i, i)$ component and in the definition of $v_{i}$. The proof is virtually the same for both cases, see Proposition 3.3 in [9]. 
(iii) From (ii) we see that if $\xi=\rho^{*}(T) I_{N}$, then

$$
t_{i i}^{2}=\frac{\chi_{\underline{m}_{i}}(T)}{\chi_{\underline{\underline{m}}_{i}}(T)}=\frac{\operatorname{det} \breve{\phi}_{i}\left(I_{N}\right)}{\operatorname{det} \phi_{i}\left(I_{N}\right)} \frac{\operatorname{det} \phi_{i}(\xi)}{\operatorname{det} \breve{\phi}_{i}(\xi)}=n_{i}^{-1} \frac{\operatorname{det} \phi_{i}(\xi)}{\operatorname{det} \breve{\phi}_{i}(\xi)} .
$$

(iv) Using the block decomposition given in (i), one has

$$
\begin{gathered}
\operatorname{tr}\left(\phi_{i}(\alpha) \phi_{i}\left(I_{N}\right)^{-1} \phi_{i}(\beta) \phi_{i}\left(I_{N}\right)^{-1}\right)-\operatorname{tr}\left(\check{\phi}_{i}(\alpha) \check{\phi}_{i}\left(I_{N}\right)^{-1} \breve{\phi}_{i}(\beta) \check{\phi}_{i}\left(I_{N}\right)^{-1}\right) \\
=\alpha_{i i} \beta_{i i}+n_{i}^{-1} \operatorname{tr}\left(v_{i}(\alpha)^{\top} \breve{\phi}_{i}\left(I_{N}\right)^{-1} v_{i}(\beta)+v_{i}(\beta)^{\top} \breve{\phi}_{i}\left(I_{N}\right)^{-1} v_{i}(\alpha)\right)
\end{gathered}
$$

and the assertion follows from the definition of $(\cdot \mid \cdot)$ and $v_{i}$.

\section{RIESZ MEASURES AND WISHART EXPONENTIAL FAMILIES}

Generalized power functions play a very important role and this is due to the following

THEOREM 4.1 ([8], [14]). (i) There exists a positive measure $\mathcal{R}_{\underline{s}}$ on $\mathcal{Z}_{\mathcal{V}}$ with the Laplace transform

$$
L_{\mathcal{R}_{\underline{s}}}(\xi)=\delta_{-\underline{s}}(\xi), \quad \xi \in \mathcal{Q} \mathcal{V},
$$

if and only if $\underline{s} \in \Xi:=\bigsqcup_{\underline{\varepsilon} \in\{0,1\}^{r}} \Xi(\underline{\varepsilon})$ (disjoint union), where

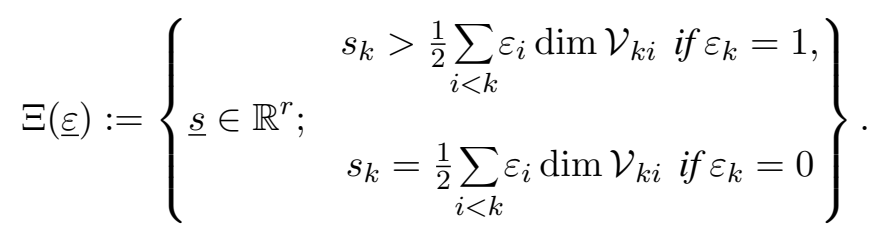

The support of $\mathcal{R}_{\underline{s}}$ is contained in $\overline{\mathcal{P}_{\mathcal{V}}}$.

(ii) There exists a positive measure $\mathcal{R}_{\underline{s}}^{*}$ on $\mathcal{Z}_{\mathcal{V}}^{*} \equiv \mathcal{Z}_{\mathcal{V}}$ with the Laplace transform

$$
L_{\mathcal{R}_{\underline{s}}^{*}}(\theta)=\Delta_{-\underline{s}}(\theta), \quad \theta \in \mathcal{P}_{\mathcal{V}},
$$

if and only if $\underline{s} \in \mathfrak{X}:=\bigsqcup_{\underline{\varepsilon} \in\{0,1\}^{r}} \mathfrak{X}(\underline{\varepsilon})$, where

$$
\mathfrak{X}(\underline{\varepsilon}):=\left\{\begin{array}{ll} 
& s_{k}>\frac{1}{2} \sum_{l>k} \varepsilon_{l} \operatorname{dim} \mathcal{V}_{l k} \text { if } \varepsilon_{k}=1, \\
\underline{s} \in \mathbb{R}^{r} ; & s_{k}=\frac{1}{2} \sum_{l>k} \varepsilon_{l} \operatorname{dim} \mathcal{V}_{l k} \text { if } \varepsilon_{k}=0
\end{array}\right\} .
$$

The support of $\mathcal{R}_{\underline{s}}^{*}$ is contained in $\overline{\mathcal{Q} \mathcal{V}}$. 
The measure $\mathcal{R}_{\underline{s}}$ (resp. $\mathcal{R}_{s}^{*}$ ) is called the Riesz measure on the cone $\mathcal{P}_{\mathcal{V}}$ (resp. $\mathcal{Q}_{\mathcal{V}}$ ). The sets $\Xi$ and $\mathfrak{X}$ are called the Gindikin-Wallach sets.

Riesz measures were described explicitly in [114]. The measure $\mathcal{R}_{\underline{s}}$ (resp. $\mathcal{R}_{s}^{*}$ ) is singular unless $\underline{s} \in \Xi(1, \ldots, 1)$ (resp. $\underline{s} \in \mathfrak{X}(1, \ldots, 1)$ ). If $\underline{s} \in \Xi(1, \ldots, 1)$ (resp. $\underline{s} \in \mathfrak{X}(1, \ldots, 1))$, then the Riesz measure is an absolutely continuous measure with respect to the Lebesgue measure. In such a case, the support of $\mathcal{R}_{\underline{s}}$ (resp. $\mathcal{R}_{\underline{s}}^{*}$ ) equals $\overline{\mathcal{P}_{\mathcal{V}}}$ (resp. $\overline{\mathcal{Q}_{\mathcal{V}}}$ ).

We are interested in the description of natural exponential families generated by $\mathcal{R}_{\underline{s}}$ and $\mathcal{R}_{\underline{s}}^{*}$. Members of $F\left(\mathcal{R}_{\underline{s}}\right)$ and $F\left(\mathcal{R}_{\underline{s}}^{*}\right)$ are called Wishart distributions on $\mathcal{P}_{\mathcal{V}}$ and $\mathcal{Q}_{\mathcal{V}}$, respectively. In order to define $\bar{N} E F$ s generated by $\mathcal{R}_{\underline{s}}$ and $\mathcal{R}_{\underline{s}}^{*}$ we have to ensure that $\mathcal{R}_{\underline{s}} \in \mathcal{M}\left(\mathcal{Z}_{\mathcal{V}}\right)$ and $\mathcal{R}_{\underline{s}}^{*} \in \mathcal{M}\left(\mathcal{Z}_{\mathcal{V}}^{*}\right)$ at least for some $\underline{s}$. We have the following

THEOREM 4.2 ([20], Theorem 3.4). (i) Let $\underline{s} \in \Xi$. The support of $\mathcal{R}_{\underline{s}}$ is not concentrated on any affine hyperplane in $\mathcal{Z}_{\mathcal{V}}$ if and only if $s_{k}>0$ for all $k=$ $1, \ldots, r$.

(ii) Let $\underline{s} \in \mathfrak{X}$. The support of $\mathcal{R}_{s}^{*}$ is not concentrated on any affine hyperplane in $\mathcal{Z}_{\mathcal{V}}^{*}$ if and only if $s_{k}>0$ for all $k=1, \ldots, r$.

4.1. Group equivariance of the Wishart exponential families. We say that a measure $\mu$ on $\mathbb{E}$ is relatively invariant under a subgroup $G$ of $G L(\mathbb{E})$ if for all $g \in$ $G$ there exists a constant $c_{g}>0$ for which $\mu(g A)=c_{g} \mu(A)$ for any measurable $A \subset \mathbb{E}$. This condition is equivalent to

$$
L_{\mu}\left(g^{*} \theta\right)=c_{g}^{-1} L_{\mu}(\theta), \quad \theta \in \Theta(\mu),
$$

where $g^{*}$ is the adjoint of $g$.

Formulas (B.2) and (B.3) imply that the Riesz measure $\mathcal{R}_{\underline{s}}$ is invariant under the group $\rho\left(H_{\mathcal{V}}\right)$, while the dual Riesz measure $\mathcal{R}_{\underline{s}}^{*}$ is invariant under $\rho^{*}\left(H_{\mathcal{V}}\right)$. It follows that the Wishart exponential family $F\left(\mathcal{R}_{\underline{s}}\right)$ is invariant under $\rho\left(H_{\mathcal{V}}\right)$ and, analogously, $F\left(\mathcal{R}_{\underline{s}}^{*}\right)$ is invariant under $\rho^{*}\left(H_{\mathcal{V}}\right)$.

\section{THE INVERSE OF THE MEAN MAP AND THE LAURITZEN FORMULA ON $\mathcal{Q} \vee$}

Let $\underline{s} \in \mathfrak{X} \cap \mathbb{R}_{>0}^{r}$. Then we have $\mathcal{R}_{s}^{*} \in \mathcal{M}\left(\mathcal{Z}_{\mathcal{V}}^{*}\right)$ by Theorem 4.2 . Denote by $\psi_{\underline{s}}:=\psi_{\mathcal{R}_{\underline{s}}^{*}}$ the inverse of the mean map from $M_{F\left(\mathcal{R}_{\underline{s}}^{*}\right)}$ to $\Theta\left(\mathcal{R}_{\underline{s}}^{*}\right)$. In this section, we give an explicit formula for $\psi_{\underline{s}}(m), m \in M_{F\left(\mathcal{R}_{s}^{*}\right)}$. Thanks to Theorem 4.1, we have $\mathcal{P}_{\mathcal{V}} \subset \Theta\left(\mathcal{R}_{\underline{s}}^{*}\right)$ and $M_{F\left(\mathcal{R}_{\underline{s}}^{*}\right)} \subset \mathcal{Q}_{\mathcal{V}}$. Applying Proposition IV.3 of [17], we can show that $\mathcal{P}_{\mathcal{V}}=\Theta\left(\mathcal{R}_{\underline{s}}^{*}\right)$ and $M_{F\left(\mathcal{R}_{\underline{s}}^{*}\right)}=\mathcal{Q}_{\mathcal{V}}$. Indeed, it suffices to check that, for any sequence $\left\{y_{k}\right\}_{k \in \mathbb{N}}$ in $\mathcal{P}_{\mathcal{V}}$ converging to a point in $\partial \mathcal{P}_{\mathcal{V}}$, we have $\lim _{k \rightarrow \infty} \Delta_{-\underline{s}}\left(y_{k}\right)=+\infty$ because $\underline{s} \in \mathbb{R}_{>0}^{r}$. 
Proposition 5.1. The inverse of the mean map on $\mathcal{Q}_{\mathcal{V}}$ is expressed by

$$
\psi_{\underline{s}}(m)=-\left(\log \delta_{-\underline{s}}\right)^{\prime}(m) .
$$

Pr o o f. By Lemma 2.$]$ we obtain $\psi_{\underline{s}}=-\left(\log J_{\mathcal{R}_{\underline{s}}^{*}}\right)^{\prime}$. For $T \in H_{\mathcal{V}}$, we have

$$
J_{\mathcal{R}_{\underline{s}}^{*}}\left(\rho^{*}(T) I_{N}\right)=\sup _{\theta \in \mathcal{P}_{\mathcal{V}}} \frac{e^{-\left\langle\rho^{*}(T) I_{N}, \theta\right\rangle}}{L_{\mathcal{R}_{\underline{s}}^{*}}(\theta)}=\sup _{\theta \in \mathcal{P}_{\mathcal{V}}} \chi_{-\underline{s}}(T) \frac{e^{-\left\langle I_{N}, \rho(T) \theta\right\rangle}}{L_{\mathcal{R}_{\underline{s}}^{*}}(\rho(T) \theta)},
$$

where the last equality follows from (B.2). Since $\rho(T) \mathcal{P}_{\mathcal{V}}=\mathcal{P}_{\mathcal{V}}$, we get

$$
J_{\mathcal{R}_{\underline{s}}^{*}}\left(\rho^{*}(T) I_{N}\right)=\chi_{-\underline{s}}(T) \cdot \sup _{\theta \in \mathcal{P}_{\mathcal{V}}} \frac{e^{-\left\langle I_{N}, \theta\right\rangle}}{L_{\mathcal{R}_{\underline{s}}^{*}}(\theta)}=\delta_{-\underline{s}}\left(\rho^{*}(T) I_{N}\right) J_{\mathcal{R}_{\underline{s}}^{*}}\left(I_{N}\right) .
$$

We see that the function $\delta_{-\underline{s}}$ equals $J_{\mathcal{R}_{\underline{s}}^{*}}$ up to a constant multiple. Therefore, $-\left(\log \delta_{-\underline{s}}\right)^{\prime}$ coincides with $-\left(\log J_{\mathcal{R}_{\underline{s}}^{*}}\right)^{\prime}$.

Let us evaluate $\psi_{\underline{s}}(m) \in \mathcal{P}_{\mathcal{V}}$ for $m \in \mathcal{Q}_{\mathcal{V}}$. In general, for a positive integer $M$, we regard the set $\operatorname{Sym}(M, \mathbb{R})$ of $M \times M$ symmetric matrices as a Euclidean vector space with the trace inner product $\operatorname{tr}(X Y)(X, Y \in \operatorname{Sym}(M, \mathbb{R}))$. Then we consider the linear map $\phi_{i}^{*}: \operatorname{Sym}\left(m_{i}, \mathbb{R}\right) \rightarrow \mathcal{Z}_{\mathcal{V}}, i=1, \ldots, r$, adjoint to $\phi_{i}$ : $\mathcal{Z}_{\mathcal{V}} \rightarrow \operatorname{Sym}\left(m_{i}, \mathbb{R}\right)$ defined in such a way that

$$
\left\langle\xi, \phi_{i}^{*}(X)\right\rangle=\operatorname{tr}\left(\phi_{i}(\xi) X\right), \quad X \in \operatorname{Sym}\left(m_{i}, \mathbb{R}\right), \xi \in \mathcal{Z}_{\mathcal{V}}
$$

The linear map $\breve{\phi}_{i}^{*}: \operatorname{Sym}\left(m_{i}-1, \mathbb{R}\right) \rightarrow \mathcal{Z}_{\mathcal{V}}, i=1, \ldots, r$, adjoint to $\check{\phi}_{i}: \mathcal{Z}_{\mathcal{V}} \rightarrow$ $\operatorname{Sym}\left(m_{i}-1, \mathbb{R}\right)$ is defined similarly.

THEOREM 5.1. The inverse of the mean map on $\mathcal{Q} v$ is given by the formula

$$
\psi_{\underline{s}}(m)=s_{r} \phi_{r}^{*}\left(\phi_{r}(m)^{-1}\right)+\sum_{i=1}^{r-1} s_{i}\left(\phi_{i}^{*}\left(\phi_{i}(m)^{-1}\right)-\breve{\phi}_{i}^{*}\left(\check{\phi}_{i}(m)^{-1}\right)\right) .
$$

Pro o f. For any $\alpha \in \mathcal{Z}_{\mathcal{V}}$, we see from (5.]) and Proposition $3 . \mathbb{1}$ (iii) that

$$
\begin{aligned}
\left\langle\alpha, \psi_{\underline{s}}(m)\right\rangle & =-D_{\alpha} \log \delta_{-\underline{s}}(m) \\
& =s_{r} D_{\alpha} \log \phi_{r}(m)+\sum_{i=1}^{r-1} s_{i} D_{\alpha}\left(\log \operatorname{det} \phi_{i}(m)-\log \operatorname{det} \breve{\phi}_{i}(m)\right),
\end{aligned}
$$

where $D_{\alpha}$ denotes the directional derivative in the direction of $\alpha$. By the wellknown formula for the derivative of log-determinant, we get

$$
\left\langle\alpha, \psi_{\underline{s}}(m)\right\rangle=s_{r} \frac{\phi_{r}(\alpha)}{\phi_{r}(m)}+\sum_{i=1}^{r-1} s_{i}\left(\operatorname{tr}\left(\phi_{i}(\alpha) \phi_{i}(m)^{-1}\right)-\operatorname{tr}\left(\check{\phi}_{i}(\alpha) \check{\phi}_{i}(m)^{-1}\right)\right) .
$$


Using the adjoint maps, we rewrite (5.3) as

$$
\begin{aligned}
\left\langle\alpha, \psi_{\underline{s}}(m)\right\rangle= & s_{r}\left\langle\alpha, \phi_{r}^{*}\left(\phi_{r}(m)^{-1}\right)\right\rangle \\
& +\sum_{i=1}^{r-1} s_{i}\left(\left\langle\alpha, \phi_{i}^{*}\left(\phi_{i}(m)^{-1}\right)\right\rangle-\left\langle\alpha, \breve{\phi}_{i}^{*}\left(\check{\phi}_{i}(m)^{-1}\right)\right\rangle\right),
\end{aligned}
$$

so that we obtain formula (5.2).

Let $\underline{n}:=\left(n_{1}, \ldots, n_{r}\right)$. Noting that $\hat{m}^{-1} \in \mathcal{P} \mathcal{V}$, we have

$$
\operatorname{det} \hat{m}^{-1}=\Delta_{\underline{n}}\left(\hat{m}^{-1}\right)=\delta_{-\underline{n}}(m)
$$

by (B.d) and (B.6). Thus, for $\alpha \in \mathcal{Z}_{\mathcal{V}}$ we observe that

$$
\left\langle\alpha, \psi_{\underline{n}}(m)\right\rangle=-D_{\alpha} \log \delta_{-\underline{n}}(m)=-D_{\alpha} \log \operatorname{det} \hat{m}^{-1}=\operatorname{tr}\left(\alpha \hat{m}^{-1}\right)=\left\langle\alpha, \hat{m}^{-1}\right\rangle .
$$

Therefore, by (15.2) we get

COROLlaRY 5.1. The inverse of the bijection $y \mapsto \pi\left(y^{-1}\right), \mathcal{P}_{\mathcal{V}} \rightarrow \mathcal{Q}_{\mathcal{V}}$ is given explicitly by

$m \mapsto \hat{m}^{-1}=\psi_{\underline{n}}(m)=n_{r} \phi_{r}^{*}\left(\phi_{r}(m)^{-1}\right)+\sum_{i=1}^{r-1} n_{i}\left(\phi_{i}^{*}\left(\phi_{i}(m)^{-1}\right)-\breve{\phi}_{i}^{*}\left(\breve{\phi}_{i}(m)^{-1}\right)\right)$.

If $n_{1}=n_{2}=\ldots=n_{r}=1$, then (5.4) yields the Lauritzen formula (5.21) in [22] for homogeneous graphical cones (cf. Example 6.11). Formula (5.4) generalizes the Lauritzen formula to all homogeneous cones.

\section{VARIANCE FUNCTION OF WISHART EXPONENTIAL FAMILIES ON $\mathcal{Q}_{\mathcal{V}}$}

As in the previous section, let $\underline{s} \in \mathfrak{X} \cap \mathbb{R}_{>0}^{r}$.

LEMMA 6.1. The variance functions of the Wishart exponential families satisfy the following identities:

$$
\begin{array}{ll}
\mathbb{V}_{F\left(\mathcal{R}_{\underline{s}}^{*}\right)}\left(\rho^{*}(T) I_{N}\right)=\rho^{*}(T) \mathbb{V}_{F\left(\mathcal{R}_{\underline{s}}^{*}\right)}\left(I_{N}\right) \rho(T), & T \in H_{\mathcal{V}}, \\
\mathbb{V}_{F\left(\mathcal{R}_{\underline{s}}\right)}\left(\rho(T) I_{N}\right)=\rho(T) \mathbb{V}_{F\left(\mathcal{R}_{\underline{s}}\right)}\left(I_{N}\right) \rho^{*}(T), & T \in H_{\mathcal{V}} .
\end{array}
$$

Pro of. The identity (6.1) for the variance function follows from the invariance of $F\left(\mathcal{R}_{\underline{s}}^{*}\right)$ under $\rho\left(H_{\mathcal{V}}\right)$ (see, for example, formula (2.2) in [20]). The invariance property of $\mathcal{R}_{\underline{s}}$ results in identity (6.2).

In [20], Theorem 7, it was shown that property (6.2) actually characterizes measure $\mathcal{R}_{\underline{s}}$. The same is true for (G.T) and $\mathcal{R}_{\underline{s}}^{*}$. 
Recall that $N=n_{1}+\ldots+n_{r}=N_{r}$. For $z \in \operatorname{Sym}\left(N_{k}, \mathbb{R}\right)$, we define the matrix $z_{0} \in \operatorname{Sym}(N, \mathbb{R})$ completed with zeros, that is, $\left(z_{0}\right)_{\{1: k\}}=z$ and $\left(z_{0}\right)_{i j}=0$ if $\max \{i, j\}>N_{k}$. Set $J_{k}:=\left(I_{N_{k}}\right)_{0} \in \mathcal{Z}_{\mathcal{V}}$ and $J_{k}^{*}:=I_{N}-J_{k}$. The proof of the following proposition is elementary and can be found in [10].

Proposition 6.1. If $y=T^{\top} T \in \operatorname{Sym}_{+}(N, \mathbb{R})$ with $T \in H_{\mathcal{V}}$, then

$$
T^{\top} J_{k}^{*} T=y-\left[\left(y^{-1}\right)_{\{1: k\}}\right]_{0}^{-1} .
$$

Now we are ready to state and prove our main theorem.

THEOREM 6.1. Let $\underline{s} \in \mathfrak{X} \cap \mathbb{R}_{>0}^{r}$. Then, the variance function of $F\left(\mathcal{R}_{s}^{*}\right)$ is given by $\left(m \in \mathcal{Q}_{\mathcal{V}}\right)$

$\mathbb{V}_{F\left(\mathcal{R}_{\underline{s}}^{*}\right)}(m)=\pi \circ\left\{\frac{n_{1}}{s_{1}} \rho(\hat{m})+\sum_{i=2}^{r}\left(\frac{n_{i}}{s_{i}}-\frac{n_{i-1}}{s_{i-1}}\right) \rho\left(\hat{m}-\left[\left(\hat{m}^{-1}\right)_{\{1: i-1\}}\right]_{0}^{-1}\right)\right\}$.

Pro of. Similarly to the proof of Theorem [.], using formula (5.]) from Proposition 5.1], we obtain

$$
\begin{aligned}
& \left\langle\alpha, \psi_{\underline{s}}^{\prime}(m) \beta\right\rangle=-D_{\alpha, \beta}^{2} \log \delta_{-\underline{s}}(m) \\
& =s_{r} D_{\alpha, \beta}^{2} \log \phi_{r}(m)+\sum_{i=1}^{r-1} s_{i}\left(D_{\alpha, \beta}^{2} \log \operatorname{det} \phi_{i}(m)-D_{\alpha, \beta}^{2} \log \operatorname{det} \breve{\phi}_{i}(m)\right) \\
& =-s_{r} \frac{\phi_{r}(\alpha) \phi_{r}(\beta)}{\phi_{r}(m)^{2}}-\sum_{i=1}^{r-1} s_{i}\left(\operatorname{tr}\left(\phi_{i}(\alpha) \phi_{i}(m)^{-1} \phi_{i}(\beta) \phi_{i}(m)^{-1}\right)\right. \\
& \left.\quad-\operatorname{tr}\left(\check{\phi}_{i}(\alpha) \breve{\phi}_{i}(m)^{-1} \breve{\phi}_{i}(\beta) \check{\phi}_{i}(m)^{-1}\right)\right),
\end{aligned}
$$

where $D_{\alpha, \beta}^{2}=D_{\alpha} D_{\beta}$. Setting $m=I_{N}$, we obtain by Proposition B.1(iv)

$$
\left\langle\alpha, \psi_{\underline{s}}^{\prime}\left(I_{N}\right) \beta\right\rangle=-s_{r} \alpha_{r r} \beta_{r r}-\sum_{i=1}^{r-1} \frac{s_{i}}{n_{i}}\left(n_{i} \alpha_{i i} \beta_{i i}+2 \sum_{l=i+1}^{r} n_{l}\left(\alpha_{l i} \mid \beta_{l i}\right)\right) .
$$

Recall that

$$
J_{k}^{*}=\left(\begin{array}{cc}
0_{N_{k}} & \\
& I_{n_{k+1}+\ldots+n_{r}}
\end{array}\right) \in \mathcal{Z}_{\mathcal{V}}
$$

and set $\mathbb{P}_{k}:=\rho\left(J_{k}^{*}\right), k=1, \ldots, r-1, \mathbb{P}_{0}=\operatorname{Id}_{\mathcal{Z}_{\mathcal{V}}} \cdot \mathbb{P}_{i}$ is the orthogonal projection onto $\bigoplus_{i<k, l \leqslant r} \mathcal{V}_{l k}$. Then, through a direct computation, one can show that for $i=1, \ldots, r-1$,

$$
n_{i} \alpha_{i i} \beta_{i i}+2 \sum_{l=i+1}^{r} n_{l}\left(\alpha_{l i} \mid \beta_{l i}\right)=\left\langle\alpha,\left(\mathbb{P}_{i-1}-\mathbb{P}_{i}\right) \beta\right\rangle
$$


and

$$
n_{r} \alpha_{r r} \beta_{r r}=\left\langle\alpha, \mathbb{P}_{r-1} \beta\right\rangle .
$$

These equalities imply that $\left(\mathbb{P}_{r}:=0_{\mathcal{Z}_{\mathcal{V}}}\right)$

$$
\psi_{\underline{s}}^{\prime}\left(I_{N}\right)=-\sum_{i=1}^{r} \frac{s_{i}}{n_{i}}\left(\mathbb{P}_{i-1}-\mathbb{P}_{i}\right)
$$

Since $\left(\mathbb{P}_{i-1}-\mathbb{P}_{i}\right)\left(\mathbb{P}_{j-1}-\mathbb{P}_{j}\right)=\delta_{i j}\left(\mathbb{P}_{i-1}-\mathbb{P}_{i}\right)$, we have

$$
\left[\psi_{\underline{s}}^{\prime}\left(I_{N}\right)\right]^{-1}=-\sum_{i=1}^{r} \frac{n_{i}}{s_{i}}\left(\mathbb{P}_{i-1}-\mathbb{P}_{i}\right) .
$$

Indeed,

$$
\psi_{\underline{s}}^{\prime}\left(I_{N}\right) \sum_{j=1}^{r}\left(-\frac{n_{j}}{s_{j}}\left(\mathbb{P}_{j-1}-\mathbb{P}_{j}\right)\right)=\sum_{i=1}^{r}\left(\mathbb{P}_{i-1}-\mathbb{P}_{i}\right)=\mathbb{P}_{0}=\operatorname{Id}_{\mathcal{Z}_{\mathcal{V}}} .
$$

Thus, (6.3) gives us

$$
\mathbb{V}_{F\left(\mathcal{R}_{\underline{s}}^{*}\right)}\left(I_{N}\right)=-\left[\psi_{\underline{s}}^{\prime}\left(I_{N}\right)\right]^{-1}=\frac{n_{1}}{s_{1}} \operatorname{Id}_{\mathcal{Z}_{\mathcal{V}}}+\sum_{i=2}^{r}\left(\frac{n_{i}}{s_{i}}-\frac{n_{i-1}}{s_{i-1}}\right) \mathbb{P}_{i-1}
$$

Finally, using (b.]), we obtain for $m=\rho^{*}(T) I_{N}$,

$$
\begin{aligned}
\mathbb{V}_{F\left(\mathcal{R}_{\underline{s}}^{*}\right)}(m) & =\rho^{*}(T) \mathbb{V}_{F\left(\mathcal{R}_{\underline{s}}^{*}\right)}\left(I_{N}\right) \rho(T) \\
& =\frac{n_{1}}{s_{1}} \rho^{*}(T) \rho(T)+\sum_{i=2}^{r}\left(\frac{n_{i}}{s_{i}}-\frac{n_{i-1}}{s_{i-1}}\right) \rho^{*}(T) \mathbb{P}_{i-1} \rho(T) .
\end{aligned}
$$

Since $\rho^{*}(T)=\pi \circ \rho\left(T^{\top}\right)$ and $\hat{m}=T^{\top} T$, we have

$$
\rho^{*}(T) \rho(T)=\pi \circ \rho\left(T^{\top}\right) \rho(T)=\pi \circ \rho\left(T^{\top} T\right)=\pi \circ \rho(\hat{m}),
$$

and, by Proposition 6.], for $i=2, \ldots, r$,

$$
\rho^{*}(T) \mathbb{P}_{i-1} \rho(T)=\pi \circ \rho\left(T^{\top} J_{i-1}^{*} T\right)=\pi \circ \rho\left(\hat{m}-\left[\left(\hat{m}^{-1}\right)_{\{1: i-1\}}\right]_{0}^{-1}\right) .
$$

EXAMPLE 6.1. Let us apply Theorem 6.1] to the Wishart exponential families on the Vinberg cone. Let

$$
\mathcal{Z}_{\mathcal{V}}:=\left\{\left(\begin{array}{ccc}
x_{11} & 0 & x_{31} \\
0 & x_{22} & x_{32} \\
x_{31} & x_{32} & x_{33}
\end{array}\right): x_{11}, x_{22}, x_{33}, x_{31}, x_{32} \in \mathbb{R}\right\} .
$$


Conditions (V1)-(V3) are satisfied and we have $n_{1}=n_{2}=n_{3}=1, N=r=3$. Then,

$$
\mathcal{P}_{\mathcal{V}}=\mathcal{Z}_{\mathcal{V}} \cap \operatorname{Sym}_{+}(3, \mathbb{R})=\left\{x \in \mathcal{Z}_{\mathcal{V}}: x_{11}>0, x_{22}>0, \operatorname{det} x>0 \in \mathbb{R}\right\}
$$

and its dual cone is given by

$$
\mathcal{Q}_{\mathcal{V}}=\left\{\xi \in \mathcal{Z}_{\mathcal{V}}: \xi_{33}>0, \xi_{11} \xi_{33}>\xi_{31}^{2}, \xi_{22} \xi_{33}>\xi_{32}^{2}\right\}
$$

The cone $\mathcal{Q}_{\mathcal{V}}$ is called the Vinberg cone, while $\mathcal{P}_{\mathcal{V}}$ is called the dual Vinberg cone. The cones $\mathcal{Q}_{\mathcal{V}}$ and $\mathcal{P}_{\mathcal{V}}$ are the lowest dimensional non-symmetric homogeneous cones.

For $\xi \in \mathcal{Z}_{\mathcal{V}}$, we have

$$
\begin{gathered}
\phi_{1}(\xi)=\left(\begin{array}{ll}
\xi_{11} & \xi_{31} \\
\xi_{31} & \xi_{33}
\end{array}\right)=: \xi_{\{1,3\}}, \quad \phi_{2}(\xi)=\left(\begin{array}{ll}
\xi_{22} & \xi_{32} \\
\xi_{32} & \xi_{33}
\end{array}\right)=: \xi_{\{2,3\}}, \\
\phi_{3}(\xi)=\check{\phi}_{1}(\xi)=\check{\phi}_{2}(\xi)=\xi_{33},
\end{gathered}
$$

so that

$$
\begin{gathered}
\phi_{1}^{*}\left(\begin{array}{ll}
a & b \\
b & c
\end{array}\right)=\left(\begin{array}{ccc}
a & 0 & b \\
0 & 0 & 0 \\
b & 0 & c
\end{array}\right), \quad \phi_{2}^{*}\left(\begin{array}{ll}
a & b \\
b & c
\end{array}\right)=\left(\begin{array}{lll}
0 & 0 & 0 \\
0 & a & b \\
0 & b & c
\end{array}\right), \\
\phi_{3}^{*}(a)=\breve{\phi}_{1}^{*}(a)=\breve{\phi}_{2}^{*}(a)=a E_{33}
\end{gathered}
$$

for $a, b, c \in \mathbb{R}$, where $E_{i i}$ is the diagonal $3 \times 3$ matrix with one in the $(i, i)$ entry and zero elsewhere. Therefore, for $m \in \mathcal{Q} \mathcal{V}$ and $\underline{s}=\left(s_{1}, s_{2}, s_{3}\right)$ we obtain by (5.2)

$$
\begin{aligned}
\psi_{\underline{s}}(m)= & s_{1}\left(\begin{array}{ccc}
\frac{m_{33}}{\left|m_{\{1,3\}}\right|} & 0 & -\frac{m_{31}}{\left|m_{\{1,3\}}\right|} \\
0 & 0 & 0 \\
-\frac{m_{31}}{\left|m_{\{1,3\}}\right|} & 0 & \frac{m_{11}}{\left|m_{\{1,3\}}\right|}
\end{array}\right)+s_{2}\left(\begin{array}{ccc}
0 & 0 & 0 \\
0 & \frac{m_{33}}{\left|m_{\{2,3\}}\right|} & -\frac{m_{32}}{\left|m_{\{2,3\}}\right|} \\
0 & -\frac{m_{32}}{\left|m_{\{2,3\}}\right|} & \frac{m_{22}}{\left|m_{\{2,3\}}\right|}
\end{array}\right) \\
& +\left(s_{3}-s_{1}-s_{2}\right) \frac{1}{m_{33}} E_{33} .
\end{aligned}
$$

In particular, (5.4) tells us that

$$
\begin{aligned}
\hat{m}^{-1}= & \left(\begin{array}{ccc}
\frac{m_{33}}{\left|m_{\{1,3\}}\right|} & 0 & -\frac{m_{31}}{\left|m_{\{1,3\}}\right|} \\
0 & 0 & 0 \\
-\frac{m_{31}}{\left|m_{\{1,3\}}\right|} & 0 & \frac{m_{33}}{\left|m_{\{1,3\}}\right|}
\end{array}\right)+\left(\begin{array}{ccc}
0 & 0 & 0 \\
0 & \frac{m_{33}}{\left|m_{\{2,3\}}\right|} & -\frac{m_{32}}{\left|m_{\{2,3\}}\right|} \\
0 & -\frac{m_{32}}{\left|m_{\{2,3\}}\right|} & \frac{m_{22}}{\left|m_{\{2,3\}}\right|}
\end{array}\right) \\
& -\left(\begin{array}{ccc}
0 & 0 & 0 \\
0 & 0 & 0 \\
0 & 0 & \frac{1}{m_{33}}
\end{array}\right),
\end{aligned}
$$


where $\left|m_{\{1,3\}}\right|=m_{11} m_{33}-m_{31}^{2}$ and $\left|m_{\{2,3\}}\right|=m_{22} m_{33}-m_{32}^{2}$. This is exactly the Lauritzen formula. Moreover, we have

$$
\hat{m}=\left(\begin{array}{ccc}
m_{11} & \frac{m_{31} m_{32}}{m_{33}} & m_{31} \\
\frac{m_{31} m_{32}}{m_{33}} & m_{22} & m_{32} \\
m_{31} & m_{32} & m_{33}
\end{array}\right)
$$

and it is easy to see that the projection $\pi: \operatorname{Sym}(3, \mathbb{R}) \rightarrow \mathcal{Z}_{\mathcal{V}}$ sets zero in the $(1,2)$ and $(2,1)$ entries, leaving all other entries unchanged.

We shall use Theorem 6.$]$ to give $\mathbb{V}_{F\left(\mathcal{R}_{\underline{s}}^{*}\right)}$ explicitly. We put $M_{i}=\frac{\left|m_{\{i, 3\}}\right|}{m_{33}} E_{i i}$ for $i=1,2$. Then we have, by (6.5),

$$
\left[\left(\hat{m}^{-1}\right)_{\{1: 1\}}\right]_{0}^{-1}=M_{1}, \quad\left[\left(\hat{m}^{-1}\right)_{\{1: 2\}}\right]_{0}^{-1}=M_{1}+M_{2} .
$$

Theorem 6.1 gives, for $\underline{s} \in \mathfrak{X} \cap \mathbb{R}_{>0}^{3}$,

$$
\begin{aligned}
\mathbb{V}_{F\left(\mathcal{R}_{\underline{s}}^{*}\right)}(m)=\pi \circ\left\{\frac{1}{s_{1}} \rho(\hat{m})\right. & +\left(\frac{1}{s_{2}}-\frac{1}{s_{1}}\right) \rho\left(\hat{m}-M_{1}\right) \\
& \left.+\left(\frac{1}{s_{3}}-\frac{1}{s_{2}}\right) \rho\left(\hat{m}-M_{1}-M_{2}\right)\right\} .
\end{aligned}
$$

Elementary properties of the quadratic operator $\rho$ and of its bilinear extension $\rho(a, b) x=\frac{1}{2}\left(a x b^{\top}+b x a^{\top}\right)$, and the fact that $\rho\left(M_{1}, M_{2}\right)=0$ on $\mathcal{Z}_{\mathcal{V}}$, imply the following formula, proven by different methods in [T]:

$$
\begin{aligned}
\mathbb{V}_{F\left(\mathcal{R}_{\underline{s}}^{*}\right)}(m)= & \pi \circ\left\{\left(\frac{1}{s_{1}}+\frac{1}{s_{2}}-\frac{1}{s_{3}}\right) \rho(\hat{m})\right. \\
& \left.+\left(\frac{1}{s_{3}}-\frac{1}{s_{1}}\right) \rho\left(\hat{m}-M_{1}\right)+\left(\frac{1}{s_{3}}-\frac{1}{s_{2}}\right) \rho\left(\hat{m}-M_{2}\right)\right\} .
\end{aligned}
$$

Note that for $\underline{s}=(p, p, p)$ with $p>\frac{1}{2}$, the variance function of $F\left(\mathcal{R}_{\underline{s}}^{*}\right)$ on the Vinberg cone is

$$
\mathbb{V}_{p}(m)=\frac{1}{p} \pi \circ \rho(\hat{m})
$$

\section{VARIANCE FUNCTION OF WISHART EXPONENTIAL FAMILIES ON $\mathcal{P}_{\mathcal{V}}$}

We are going to find the variance function of the NEF generated by $\mathcal{R}_{\underline{s}}$ on the cone $\mathcal{P}_{\mathcal{V}}$. Using a similar approach (see (6.4)) to that in the proof of Theorem 6.1, one can show the following proposition.

Proposition 7.1. Let $\underline{s} \in \Xi \cap \mathbb{R}_{>0}^{r}$. For any $T \in H_{\mathcal{V}}$ one has

$$
\mathbb{V}_{F\left(\mathcal{R}_{\underline{s}}\right)}\left(\rho(T) I_{N}\right)=\frac{n_{r}}{s_{r}} \rho(T) \rho^{*}(T)+\sum_{k=1}^{r-1}\left(\frac{n_{k}}{s_{k}}-\frac{n_{k+1}}{s_{k+1}}\right) \rho(T) \rho\left(J_{k}\right) \rho^{*}(T) .
$$


Pro of. We have $\Theta\left(\mathcal{R}_{\underline{s}}\right)=\mathcal{Q}_{\mathcal{V}}$ and $M_{F\left(\mathcal{R}_{s}\right)}=\mathcal{P}_{\mathcal{V}}$. By definition, $m_{\mathcal{R}_{\underline{s}}}(\theta)=$ $-\left(\log \delta_{-\underline{s}}\right)^{\prime}(\theta), \theta \in \mathcal{Q}_{\mathcal{V}}$. Use Lemma $2 . \prod$ to show that $\psi_{\mathcal{R}_{s}}(m)=-\left(\log \Delta_{-\underline{s}}\right)^{\prime}(m)$, $m \in \mathcal{P} \mathcal{V}$, and proceed as in the proof of Theorem 6.1.

Now we will also use another approach to this problem. We will use the duality of the cones $\mathcal{P}_{\mathcal{V}}$ and $\mathcal{Q}_{\mathcal{V}}$ and a matrix realization of $\mathcal{Q}_{\mathcal{V}}$. The objective is to boil down to the results of the preceding section and apply the formula for the variance function from Theorem 6.1.

The dual cone $\mathcal{Q}_{\mathcal{V}}$ to a homogeneous cone is also homogeneous. Thus, due to [15], Theorem D, it admits (under suitable linear isomorphism) a matrix realization. There exists a family $\widetilde{\mathcal{V}}=\left\{\widetilde{\mathcal{V}}_{l k}\right\}_{1 \leqslant k<l \leqslant \tilde{r}}$ satisfying (V1)-(V3) and a linear isomorphism $l: \mathcal{Z}_{\widetilde{\mathcal{V}}} \rightarrow \mathcal{Z}_{\mathcal{V}}$ such that $\mathcal{Q}_{\mathcal{V}}=l\left(\mathcal{P}_{\tilde{\mathcal{V}}}\right)$. It can be shown that $\tilde{r}=r$.

Since $l\left(\mathcal{P}_{\tilde{\mathcal{V}}}\right)=\mathcal{Q}_{\mathcal{V}}$ and $l$ is an isomorphism, for any $T \in H_{\mathcal{V}}$ there exists a unique $\widetilde{T} \in \mathrm{H}_{\widetilde{\mathcal{V}}}$ such that

$$
l\left(\rho(\widetilde{T}) I_{\tilde{N}}^{\widetilde{\mathcal{V}}}\right)=\rho^{*}(T) I_{N}^{\mathcal{V}}
$$

The linear isomorphism $l$ can be taken in such a way that if $T$ has diagonal $\left(t_{11}, \ldots, t_{r r}\right)$, then $\widetilde{T}$ has diagonal $\left(t_{r r}, \ldots, t_{11}\right)$ (see the choice of the permutation $w$ in Proposition [.4). In such a case we have $\chi_{\underline{s}}^{\mathcal{V}}(\widetilde{T})=\chi_{\underline{s}^{*}}^{\widetilde{\mathcal{V}}^{*}}(T)$, where $\underline{s}^{*}=\left(s_{r}, \ldots, s_{1}\right)$. This implies the following proposition:

PROPOSITION 7.2. There exists a family $\widetilde{\mathcal{V}}=\left\{\widetilde{\mathcal{V}}_{l k}\right\}_{1 \leqslant k<l \leqslant r}$ satisfying (V1)(V3) and a linear isomorphism $l: \mathcal{Z}_{\widetilde{\mathcal{V}}} \rightarrow \mathcal{Z}_{\mathcal{V}}$ such that $\mathcal{Q}_{\mathcal{V}}=l\left(\mathcal{P}_{\widetilde{\mathcal{V}}}\right)$ and

$$
\Delta_{\underline{s}^{*}}^{\widetilde{\mathcal{V}}}(x)=\delta_{\underline{s}}^{\mathcal{V}}(l(x)), \quad x \in \mathcal{P}_{\widetilde{\mathcal{V}}} .
$$

In this case, $\underline{s} \in \Xi_{\mathcal{V}}$ if and only if $\underline{s}^{*} \in \mathfrak{X}_{\widetilde{\mathcal{V}}}$.

The adjoint map $l^{*}: \mathcal{Z}_{\mathcal{V}} \rightarrow \mathcal{Z}_{\widetilde{\mathcal{V}}}^{*} \equiv \mathcal{Z}_{\widetilde{\mathcal{V}}}$ is a linear isomorphism such that $l^{*}\left(\mathcal{P}_{\mathcal{V}}\right)=\mathcal{Q}_{\widetilde{\mathcal{V}}}$ and

$$
\Delta_{\underline{s}}^{\mathcal{V}}(x)=\delta_{\underline{s}^{*}}^{\widetilde{\mathcal{V}}}\left(l^{*}(x)\right), \quad x \in \mathcal{P} \mathcal{V} .
$$

Consider the Riesz measure $\mathcal{R}_{\underline{s}}$ on $\mathcal{P} \mathcal{V}$. Then for any $\xi \in \mathcal{Q}_{\mathcal{V}}$

$$
L_{\mathcal{R}_{\underline{s}}}(\xi)=\delta_{-\underline{s}}^{\mathcal{V}}(\xi)=\Delta_{\underline{s}^{*}}^{\widetilde{\mathcal{V}}}\left(\left(l^{*}\right)^{-1}(\xi)\right)=L_{\mathcal{R}_{\underline{s}^{*}}^{*}}\left(\left(l^{*}\right)^{-1}(\xi)\right),
$$

where $L_{\mathcal{R}_{s^{*}}^{*}}$ is the Laplace transform of the Riesz measure on $\mathcal{P}_{\widetilde{V}}$. We have proved the following

THEOREM 7.1. Let $\underline{s} \in \Xi \cap \mathbb{R}_{>0}^{r}$. Then the variance function of $F\left(\mathcal{R}_{\underline{s}}\right)$ is given by

$$
\mathbb{V}_{F\left(\mathcal{R}_{\underline{s}}\right)}(\theta)=\left(l^{*}\right)^{-1} \circ \mathbb{V}_{F\left(\mathcal{R}_{\underline{s}^{*}}^{*}\right)}\left(l^{*}(\theta)\right) \circ l^{-1}, \quad \theta \in M_{F\left(\mathcal{R}_{\underline{s}}\right)}=\mathcal{P}_{\mathcal{V}}
$$


Here $\mathbb{V}_{F\left(\mathcal{R}_{\underline{s}^{*}}^{*}\right)}$ is the variance function of the Riesz measure defined on $\mathcal{P}_{\widetilde{\mathcal{V}}}$, which can be written by using Theorem 6.1. The drawback of this result is that the map $l$, and so $l^{*}$, is generically not explicit. In the second part of this section we propose a practical construction of a matrix realization of the cone $\mathcal{Q} \mathcal{V}$. Consequently, the maps $l$ and $l^{*}$ will be available and Theorem $[$.$] will become useful in$ statistical practice.

7.1. Matrix realization of the cone $\mathcal{Q}_{\mathcal{V}}$. One general way to get a matrix realization of the cone $\mathcal{Q}_{\mathcal{V}}$ as $\mathcal{P}_{\widetilde{\mathcal{V}}}$ is as follows. Recall that $m_{i}=\operatorname{dim} W_{i}$, which was defined in Section 3.3. Noting that $\sum_{i=1}^{r} m_{i}$ equals the dimension $d$ of the cone $\mathcal{Q}_{\mathcal{V}}$, we define a linear map

$$
\Phi: \mathcal{Z}_{\mathcal{V}} \ni \xi \mapsto \operatorname{diag}\left(\phi_{1}(\xi), \phi_{2}(\xi), \ldots, \phi_{r}(\xi)\right) \in \operatorname{Sym}(d, \mathbb{R}),
$$

where $\operatorname{diag}\left(A_{1}, \ldots, A_{m}\right) \in \operatorname{Mat}\left(\sum_{k=1}^{m} n_{k}, \sum_{k=1}^{m} n_{k} ; \mathbb{R}\right)$ denotes the square matrix with matrices $A_{k} \in \operatorname{Mat}\left(n_{k}, n_{k} ; \mathbb{R}\right)$ as diagonal blocks. Put

$$
\mathcal{A}_{\mathcal{V}}:=\left\{\Phi\left(I_{N}\right)^{-1 / 2} \Phi(\xi) \Phi\left(I_{N}\right)^{-1 / 2} \xi \in \mathcal{Z}_{\mathcal{V}}\right\} .
$$

PROPOSITION 7.3. By an appropriate permutation of rows and columns, the subspace $\mathcal{A}_{\mathcal{V}}$ of $\operatorname{Sym}(d, \mathbb{R})$ gives a matrix realization of $\mathcal{Q} \mathcal{V}$, thus we have $l^{-1}=$ $\rho\left(w \Phi\left(I_{d}\right)^{-1 / 2}\right) \circ \Phi$ for some permutation matrix $w \in G L(d, \mathbb{R})$.

The permutation giving a matrix realization in Proposition 7.3 is not unique. We shall present a practical method of finding such a permutation. For $k=1, \ldots, r$, we put $\nu_{k}:=1+\sum_{i<k} \operatorname{dim} \mathcal{V}_{k i}$.

Proposition 7.4. Let $w \in G L(d, \mathbb{R})$ be a permutation matrix such that

$$
\rho\left(w \Phi\left(I_{N}\right)^{-1 / 2}\right) \Phi(\xi)=\operatorname{diag}\left(\xi_{r r} I_{\nu_{r}}, \xi_{r-1, r-1} I_{\nu_{r-1}}, \ldots, \xi_{11} I_{\nu_{1}}\right)
$$

holds for any diagonal $\xi \in \mathcal{Z}_{\mathcal{V}}$. Then $\rho(w) \mathcal{A}_{\mathcal{V}}=\mathcal{Z}_{\widetilde{\mathcal{V}}}$ with some vector spaces $\widetilde{\mathcal{V}}_{l k} \subset \operatorname{Mat}\left(\nu_{l}, \nu_{k}, \mathbb{R}\right)(1 \leqslant k<l \leqslant r)$ which satisfy (V1)-(V3).

The proofs of Propositions 7.3 and 7.4 are technical and omitted. They are available in the full version of the article [10].

Let us note that condition ([.2.) is rather easy to check, since the matrix $\rho\left(\Phi\left(I_{N}\right)^{-1 / 2}\right) \Phi(\xi)$ is diagonal if $\xi$ is diagonal.

We remark that some $\phi_{i}$ 's can be omitted from $\Phi$ : this is the "optimization" of matrix realizations of homogeneous cones, recently developed by Yamasaki and Nomura [26], see Example [T] below.

EXAMPLE 7.1. Consider the same space $\mathcal{Z}_{\mathcal{V}}$ as in Example 6.]. The variance function of a Wishart family on $\mathcal{Q} \mathcal{V}$ was derived in Example 6.1. The objective of Example Z.] is to give the variance function of a Wishart family on $\mathcal{P} \mathcal{V}$. We start with constructing a convenient matrix realization of $\mathcal{Q} \mathcal{V}$, using Propositions T.2-Z.4. 
For the dual Vinberg cone, the map $\Phi$ is defined as follows (see [17]):

$$
\left(\begin{array}{lll}
\xi_{1} & & \xi_{4} \\
& \xi_{2} & \xi_{5} \\
\xi_{4} & \xi_{5} & \xi_{3}
\end{array}\right) \rightarrow \operatorname{diag}\left(\left(\begin{array}{ll}
\xi_{1} & \xi_{4} \\
\xi_{4} & \xi_{3}
\end{array}\right),\left(\begin{array}{ll}
\xi_{2} & \xi_{5} \\
\xi_{5} & \xi_{3}
\end{array}\right),\left(\xi_{3}\right)\right)
$$

Since $\Phi\left(I_{3}\right)=I_{5}$ in this case, $\mathcal{A}_{\mathcal{V}}^{0}$ is the set of matrices of the form

$$
\operatorname{diag}\left(\left(\xi_{3}\right),\left(\begin{array}{ll}
\xi_{3} & \xi_{5} \\
\xi_{5} & \xi_{2}
\end{array}\right),\left(\begin{array}{ll}
\xi_{3} & \xi_{4} \\
\xi_{4} & \xi_{1}
\end{array}\right)\right)
$$

and applying Proposition Z.4, we obtain a matrix realization of $\mathcal{Q}_{\mathcal{V}}$ on the space $\mathcal{Z}_{\widetilde{\mathcal{V}}}$ of the matrices of the form

$$
\left(\begin{array}{ccccc}
\xi_{3} & & & & \\
& \xi_{3} & & \xi_{5} & \\
& & \xi_{3} & & \xi_{4} \\
& \xi_{5} & & \xi_{2} & \\
& & \xi_{4} & & \xi_{1}
\end{array}\right) .
$$

On the other hand, an optimal matrix realization is obtained by omitting the first diagonal $\xi_{3}$ in ([.3). In order to give the variance function for Wishart families on the dual Vinberg cone $\mathcal{P}_{\mathcal{V}}$, we will use this optimal matrix realization. The isomorphism $l: \mathcal{Z}_{\widetilde{\mathcal{V}}} \rightarrow \mathcal{Z}_{\mathcal{V}}$ is then such that

$$
l^{-1}: \mathcal{Z}_{\mathcal{V}} \ni\left(\begin{array}{ccc}
\xi_{1} & & \xi_{4} \\
& \xi_{2} & \xi_{5} \\
\xi_{4} & \xi_{5} & \xi_{3}
\end{array}\right) \rightarrow\left(\begin{array}{cccc}
\xi_{3} & & \xi_{5} & \\
& \xi_{3} & & \xi_{4} \\
\xi_{5} & & \xi_{2} & \\
& \xi_{4} & & \xi_{1}
\end{array}\right) \in \mathcal{Z}_{\widetilde{\mathcal{V}}}
$$

It is easy to verify by direct computation that $\delta_{\underline{s}}^{\mathcal{V}}(l(x))=\Delta_{s^{*}} \widetilde{\mathcal{V}}^{*}(x)$ for any $x \in \mathcal{P}_{\widetilde{\mathcal{V}}}$, where $\underline{s}^{*}=\left(s_{r}, \ldots, s_{1}\right)$. This relation is ensured by formula (ZZ.T). The adjoint $\operatorname{map} l^{*}: \mathcal{Z}_{\mathcal{V}} \rightarrow \mathcal{Z}_{\widetilde{\mathcal{V}}}$ is, for $\theta \in \mathcal{Z}_{\mathcal{V}}$,

$l^{*}\left(\begin{array}{ccc}\theta_{1} & & \theta_{4} \\ & \theta_{2} & \theta_{5} \\ \theta_{4} & \theta_{5} & \theta_{3}\end{array}\right)=\left(\begin{array}{cccc}\frac{\theta_{3}}{2} & & \theta_{5} & \\ & \frac{\theta_{3}}{2} & & \theta_{4} \\ \theta_{5} & & \theta_{2} & \\ & \theta_{4} & & \theta_{1}\end{array}\right),\left(l^{*}\right)^{-1}\left(\begin{array}{cccc}x & & u & \\ & x & & v \\ u & & y & \\ & v & & z\end{array}\right)=\left(\begin{array}{ccc}z & 0 & v \\ 0 & y & u \\ v & u & 2 x\end{array}\right)$.

For $\mathcal{Z}_{\widetilde{\mathcal{V}}}$ we have $\tilde{N}=4, \tilde{r}=3, \tilde{n}_{1}=2$ and $\tilde{n}_{2}=\tilde{n}_{3}=1$. Theorems $\mathbb{Z T}$ and 6.] imply that for $\underline{s} \in \Xi$ and $\theta \in \mathcal{P}_{\mathcal{V}}$

$$
\mathbb{V}_{F\left(\mathcal{R}_{\underline{s}}\right)}(\theta)=\left(l^{*}\right)^{-1} \circ \mathbb{V}_{F\left(\mathcal{R}_{\underline{s}^{*}}^{*}\right)}\left(l^{*}(\theta)\right) \circ l^{-1},
$$


where $\mathcal{R}_{\underline{s}^{*}}^{*}$ is the Riesz measure defined on $\mathcal{P}_{\widetilde{\mathcal{V}}}$, and for $m \in \mathcal{Q}_{\widetilde{\mathcal{V}}}$,

$$
\begin{aligned}
\mathbb{V}_{F\left(\mathcal{R}_{\underline{s}^{*}}^{*}\right.}(m)=\pi \circ\left\{\frac{2}{s_{3}} \rho(\hat{m})\right. & +\left(\frac{1}{s_{2}}-\frac{2}{s_{3}}\right) \rho\left(\hat{m}-M_{1}\right) \\
& \left.+\left(\frac{1}{s_{1}}-\frac{1}{s_{2}}\right) \rho\left(\hat{m}-M_{1,2}\right)\right\},
\end{aligned}
$$

with $M_{1}=\left[\left(\hat{m}^{-1}\right)_{\{1: 1\}}\right]_{0}^{-1}$ and $M_{1,2}=\left[\left(\hat{m}^{-1}\right)_{\{1: 2\}}\right]_{0}^{-1}$. Recall that $\{1: i\}$ notation corresponds to blocks of $\mathcal{Z}_{\widetilde{\mathcal{V}}}$, thus $\{1: 1\}=\{1,2\}$ and $\{1: 2\}=\{1,2,3\}$. The projection $\pi: \operatorname{Sym}(4, \mathbb{R}) \rightarrow \mathcal{Z}_{\widetilde{\mathcal{V}}}$ is given by

$$
\pi:\left(\begin{array}{llll}
x_{11} & x_{21} & x_{31} & x_{41} \\
x_{21} & x_{22} & x_{32} & x_{42} \\
x_{31} & x_{32} & x_{33} & x_{43} \\
x_{41} & x_{42} & x_{43} & x_{44}
\end{array}\right) \rightarrow\left(\begin{array}{cccc}
\frac{x_{11}+x_{22}}{2} & & x_{31} & \\
& \frac{x_{11}+x_{22}}{2} & & x_{42} \\
x_{31} & & x_{33} & \\
& x_{42} & & x_{44}
\end{array}\right)
$$

For

$$
m=\left(\begin{array}{cccc}
m_{3} & & m_{5} & \\
& m_{3} & & m_{4} \\
m_{5} & & m_{2} & \\
& m_{4} & & m_{1}
\end{array}\right)
$$

we have

$$
\hat{m}=\left(\begin{array}{cccc}
m_{3}-c & & m_{5} & \\
& m_{3}+c & & m_{4} \\
m_{5} & & m_{2} & \\
& m_{4} & & m_{1}
\end{array}\right)
$$

with $c=\frac{1}{2}\left(m_{4}^{2} / m_{1}-m_{5}^{2} / m_{2}\right)$. Explicit formulas for matrices $M_{1}$ and $M_{1,2}$ can be easily found. In particular, for $\underline{s}=(p, p, p)$ one has

$$
\mathbb{V}_{F\left(\mathcal{R}_{\underline{\underline{s}}}\right)}(\theta)=\frac{1}{p}\left(l^{*}\right)^{-1} \circ \pi \circ\left[2 \rho(\hat{m})-\rho\left(\hat{m}-M_{1}\right)\right] \circ l^{-1},
$$

where $m=l^{*}(\theta)$ and $\theta \in \mathcal{P}_{\mathcal{V}}$. The last formula, compared with (6.8), confirms the fact that analysis of Wishart laws on homogeneous cones $\mathcal{P}_{\mathcal{V}}$ is technically more difficult than on the cones $\mathcal{Q} \mathcal{V}$.

\section{APPLICATIONS}

8.1. Classical Wishart families $F\left(\mu_{p}\right)$ on $\operatorname{Sym}_{+}(n, \mathbb{R})$. In this case, $\mathcal{Z}_{\mathcal{V}}=$ $\operatorname{Sym}(r, \mathbb{R}), \mathcal{Q}_{\mathcal{V}}=\operatorname{Sym}_{+}(n, \mathbb{R})$, all $n_{i}=1$, the projection $\pi$ is the identity map from $\operatorname{Sym}(n, \mathbb{R})$ to itself and $\hat{m}=m$. We have $\underline{s}=p \mathbf{1}$, the measure $\mu_{p}$ is the Riesz measure $\mathcal{R}_{\underline{s}}^{*}$ and $\underline{s} \in \mathfrak{X}$ if and only if $p \in \Lambda$. Formula (ㅍ. fl) from the Introduction is instantly recovered by using Theorem 6.1 . 
8.2. The Wishart families on symmetric cones indexed by $\underline{s} \in \mathbb{R}^{r}$. The cone $\operatorname{Sym}_{+}(n, \mathbb{R})$ is the prime example of a symmetric cone, that is, a homogeneous cone $\Omega$ which is self-dual $\left(\Omega^{*}=\Omega\right)$.

The matrix realization of homogeneous cones (see Section 3. I) does not coincide with the usual setting in which symmetric cones are considered, that is, Jordan algebras, except for $\operatorname{Sym}_{+}(n, \mathbb{R})$ cone. This is the reason why there is no "automatic" correspondence between formulas for variance functions in these two settings. However, the techniques developed in this article apply in the symmetric cone setting.

Here we use the standard notation of [7]. Let $V$ be a simple Euclidean Jordan algebra of rank $r$ and let $\Omega$ be its associated irreducible symmetric cone. If $c$ is an idempotent in $V$, we denote by $V(c, 1)$ the eigenspace corresponding to the eigenvalue one of the linear operator $\mathbb{L}(x)$ on $V$, which is defined using the Jordan product $\mathbb{L}(x)=x y$. For a fixed Jordan frame $\mathbf{c}=\left(c_{1}, \ldots, c_{r}\right)$ define subspaces $V^{(k)}=V\left(c_{1}+\ldots+c_{k}, 1\right)$ and $W^{(k)}=V\left(c_{r-k+1}+\ldots+c_{r}, 1\right)$. Denote by $P_{k}$ and $P_{k}^{*}$ the orthogonal projections of $V$ onto $V^{(k)}$ and $W^{(k)}$, respectively. Let $\Delta_{\underline{s}}$ be the generalized power function with respect to $\mathbf{c}$ and $\mathbb{P}$ be the quadratic representation of $\Omega$. We consider natural exponential families generated by the Riesz measure $\mathcal{R}_{s}^{*}$ with the Laplace transform $\Delta_{-\underline{s}}$ and $\mathcal{R}_{\underline{s}}$ with the Laplace transform $\theta \mapsto \Delta_{\underline{s}}\left(\theta^{-1}\right)$. Using the same techniques as in Theorem 6.1 , but in the Euclidean Jordan algebra framework, we prove the following

Proposition 8.1. For $m \in \Omega=\Omega^{*}$,

$$
\begin{aligned}
& \mathbb{V}_{F\left(\mathcal{R}_{\underline{s}}^{*}\right)}(m)=\frac{1}{s_{1}} \mathbb{P}(m)+\sum_{i=2}^{r}\left(\frac{1}{s_{i}}-\frac{1}{s_{i-1}}\right) \mathbb{P}\left(m-\left[P_{i-1} m^{-1}\right]_{0}^{-1}\right), \\
& \mathbb{V}_{F\left(\mathcal{R}_{\underline{s}}\right)}(m)=\frac{1}{s_{r}} \mathbb{P}(m)+\sum_{k=1}^{r-1}\left(\frac{1}{s_{k}}-\frac{1}{s_{k+1}}\right) \mathbb{P}\left(m-\left[P_{r-k}^{*} m^{-1}\right]_{0}^{-1}\right),
\end{aligned}
$$

where $[\cdot]_{0}$ denotes the inclusion map from the subalgebras $V^{(k)}$ and $W^{(k)}$ to $V$.

8.3. Graphical homogeneous cones. Let $G=(V, E)$ be an undirected graph, where $V=\{1, \ldots, r\}$ is the set of vertices and $E \subset V \times V$ is the set of undirected edges, that is, if $(i, j) \in E$, then $(j, i) \in E, i, j \in V$. For statisticians, the parameter space of interest for covariance graph models is the cone $P_{G}$ of positive definite matrices with fixed zeros corresponding to the missing edges of $G$. More precisely, if

$$
\mathcal{Z}_{\mathrm{G}}:=\left\{\left(x_{i j}\right) \in \operatorname{Sym}(r, \mathbb{R}): x_{i j}=0 \text { if }(i, j) \notin E\right\},
$$

then $P_{G}$ is defined by

$$
P_{G}:=\mathcal{Z}_{\mathrm{G}} \cap \operatorname{Sym}_{+}(r, \mathbb{R}) .
$$

It is known that the cone $P_{G}$ is homogeneous if and only if $G$ is decomposable (chordal) and does not contain the graph $\bullet-\bullet-\bullet-\bullet$, denoted by $A_{4}$, as an 
induced subgraph (for details see [24], [16]). For $1 \leqslant k<l \leqslant r$, we set $\mathcal{V}_{l k}:=\mathbb{R}$ if $(k, l) \in E$, and $\mathcal{V}_{l k}:=\{0\}$ otherwise. Then it can be shown that (possibly after renumeration of vertices) the family $\left\{\mathcal{V}_{l k}\right\}_{1 \leqslant k<l \leqslant r}$ satisfies (V1)-(V3). One sees (cf. [16]) that $\mathcal{P}_{\mathcal{V}}$ is a graphical cone if and only if $n_{1}=\ldots=n_{r}=1$. Thus the trace inner product coincides with the standard inner product.

The results of the present paper apply to homogeneous graphical cones. However, in the present paper, $\mathcal{Z}_{\mathcal{V}}^{*}$ was identified with $\mathcal{Z}_{\mathcal{V}}$, whereas in the statistical approach to graphical cones one proceeds as follows.

Let $I_{G}$ be the real linear space of $G$-incomplete symmetric matrices, that is, functions $(i, j) \mapsto x_{i j}$ from $E$ to $\mathbb{R}$ such that $x_{i j}=x_{j i}$. The dual space $\mathcal{Z}_{G}^{*}$ is identified with $I_{G}$ through $\langle y, x\rangle=\sum_{(i, j) \in E} x_{i j} y_{i j},(x, y) \in \mathcal{Z}_{\mathrm{G}} \times I_{G}$, and the dual cone is denoted by $Q_{G}:=\left\{y \in I_{G}:\langle y, x\rangle>0 \forall x \in \overline{P_{G}} \backslash\{0\}\right\}$.

Let $\pi: Z_{G} \rightarrow I_{G}$ be such that $\pi(x)_{i j}=x_{i j}$ for any $(i, j) \in E$. For any $m \in$ $Q_{G}$ there exists a unique $\hat{m} \in \operatorname{Sym}_{+}(r, \mathbb{R})$ such that for all $(i, j) \in E$ one has $\hat{x}_{i j}=x_{i j}$ and such that $\hat{x}^{-1} \in P_{G}$ (see [24], p. 1279). The last definitions of $\pi$ and $\hat{m}$ on graphical cones agree with the ones given in Definitions 3.2 and 3.3. One can check (see [19]) that the function $Q_{G} \ni \eta \mapsto H(\alpha, \beta, \eta)$ considered in [24] equals the generalized power function $\delta_{\underline{s}}$ for some $\underline{s} \in \mathbb{R}^{r}$, by comparing formula (B.7) with the definition of $H(\alpha, \beta, \eta)$.

Thus Theorem 6.1] applies to the cone $Q_{G} \subset I_{G}$ with $n_{i}=1$.

Similarly, by formula (B.5), the function $P_{G} \ni y \mapsto H\left(\alpha, \beta, \pi\left(y^{-1}\right)\right)$ introduced in [24] on the cone $P_{G}$ coincides with the generalized power function $\Delta_{\underline{s}}$ for some $\underline{s}$ and the results of Section $\square$ apply to the cone $P_{G}$.

8.4. Non-homogeneous graphical cones. Recently, the variance function was also computed for the cones $Q_{G}$ corresponding to non-homogeneous graphs $G=$ $A_{n}, n \geqslant 4$, see [11]. The techniques are partly the same, but the lack of an analogue of the equivariance formula (6.1) must be overcome.

\section{REFERENCES}

[1] S. A. Andersson and T. Klein, On Riesz and Wishart distributions associated with decomposable undirected graphs, J. Multivariate Anal. 101 (4) (2010), pp. 789-810.

[2] S. A. Andersson and G. G. Wojnar, Wishart distributions on homogeneous cones, J. Theoret. Probab. 17 (4) (2004), pp. 781-818.

[3] O. Barndorff-Nielsen, Information and Exponential Families in Statistical Theory, Wiley, Chichester 2014.

[4] I. Boutouria, Characterization of the Wishart distributions on homogeneous cones, C. R. Math. Acad. Sci. Paris 341 (1) (2005), pp. 43-48.

[5] I. Boutouria and A. Hassairi, Riesz exponential families on homogeneous cones, arXiv:0906.1892v1 (2009).

[6] P. Diaconis and D. Ylvisaker, Conjugate priors for exponential families, Ann. Statist. 7 (2) (1979), pp. 269-281.

[7] J. Faraut and A. Korányi, Analysis on Symmetric Cones, The Clarendon Press, Oxford University Press, New York 1994. 
[8] S. G. Gindikin, Invariant generalized functions in homogeneous domains, Funktsional. Anal. i Prilozhen. 9 (1) (1975), pp. 56-58.

[9] P. Graczyk and H. Ishi, Riesz measures and Wishart laws associated to quadratic maps, J. Math. Soc. Japan 66 (1) (2014), pp. 317-348.

[10] P. Graczyk, H. Ishi, and B. Kołodziejek, Wishart laws and variance function on homogeneous cones, arXiv:1802.02352 (2018).

[11] P. Graczyk, H. Ishi, and S. Mamane, Wishart exponential families on cones related to tridiagonal matrices, Ann. Inst. Statist. Math. 71 (2) (2019), pp. 439-471.

[12] A. Hassairi and S. Lajmi, Riesz exponential families on symmetric cones, J. Theoret. Probab. 14 (4) (2001), pp. 927-948.

[13] T. Hastie, R. Tibshirani, and M. Wainwright, Statistical Learning with Sparsity: The Lasso and Generalizations, CRC Press, Boca Raton, FL, 2015.

[14] H. Ishi, Positive Riesz distributions on homogeneous cones, J. Math. Soc. Japan 52 (1) (2000), pp. 161-186.

[15] H. Ishi, On symplectic representations of normal j-algebras and their application to Xu's realizations of Siegel domains, Differential Geom. Appl. 24 (6) (2006), pp. 588-612.

[16] H. Ishi, On a class of homogeneous cones consisting of real symmetric matrices, Josai Math. Monogr. 6 (2013), pp. 71-80.

[17] H. Is hi, Homogeneous cones and their applications to statistics, in: Modern Methods of Multivariate Statistics, P. Graczyk and A. Hassairi (Eds.), Hermann, Paris 2014, pp. 135-154.

[18] H. Ishi, Matrix realization of homogeneous cones, Lecture Notes in Comput. Sci. Proceedings 9389 (2015), pp. 248-256.

[19] H. Ishi, Explicit formula of Koszul-Vinberg characteristic functions for a wide class of regular convex cones, Entropy 18 (11) (2016), 383.

[20] H. Ishi and B. Kołodziejek, Characterization of the Riesz exponential family on homogeneous cones, Colloq. Math. 158 (2019), pp. 45-57.

[21] K. Khare and B. Rajaratnam, Wishart distributions for decomposable covariance graph models, Ann. Statist. 39 (1) (2011), pp. 514-555.

[22] S. L. Lauritzen, Graphical Models, The Clarendon Press, Oxford University Press, New York 1996.

[23] G. Letac, A characterization of the Wishart exponential families by an invariance property, J. Theoret. Probab. 2 (1) (1989), pp. 71-86.

[24] G. Letac and H. Massam, Wishart distributions for decomposable graphs, Ann. Statist. 35 (3) (2007), pp. 1278-1323.

[25] B. Vinberg, The theory of homogeneous convex cones, Trudy Moskov. Mat. Obshch. 12 (1963), pp. 303-358.

[26] T. Yamasaki and T. Nomura, Realization of homogeneous cones through oriented graphs, Kyushu J. Math. 69 (1) (2015), pp. 11-48.

Piotr Graczyk

Laboratoire de Mathématiques LAREMA

Université d'Angers

2 Boulevard Lavoisier, 49045 Angers Cedex 01

France

E-mail: graczyk@univ-angers.fr

Bartosz Kołodziejek

Faculty of Mathematics and Information Science

Warsaw University of Technology

Koszykowa 75, 00-662 Warsaw, Poland

E-mail: b.kolodziejek@mini.pw.edu.pl
Hideyuki Ishi

Graduate School of Mathematics

Nagoya University

Furo-cho, Nagoya 464-8602, Japan JST, PRESTO, 4-1-8 Honcho Kawaguchi 332-0012, Japan

E-mail: hideyuki@math.nagoya-u.ac.jp

Received on 10.11.2017;

revised version on 30.4.2018 